\title{
New insights into Rossby wave packet properties in the extratropical UTLS using GNSS radio occultations
}

\author{
Robin Pilch Kedzierski ${ }^{1}$, Katja Matthes ${ }^{1,2}$, and Karl Bumke ${ }^{1}$ \\ ${ }^{1}$ Marine Meteorology Department, GEOMAR Helmholtz Centre for Ocean Research Kiel, Kiel, Germany \\ ${ }^{2}$ Faculty of Mathematics and Natural Sciences, Christian-Albrechts-Universität zu Kiel, Kiel, Germany \\ Correspondence: Robin Pilch Kedzierski (rpilch@geomar.de)
}

Received: 12 February 2020 - Discussion started: 4 March 2020

Revised: 15 July 2020 - Accepted: 20 August 2020 - Published: 12 October 2020

\begin{abstract}
The present study describes Rossby wave packet (RWP) properties in the upper troposphere and lower stratosphere (UTLS) with the use of Global Navigation Satellite System radio occultation (GNSS-RO) measurements. This global study covering both hemispheres' extratropics is the first to tackle medium- and synoptic-scale waves with GNSSRO. We use 1 decade of GNSS-RO temperature and pressure data from the CHAMP, COSMIC, GRACE, MetopA, Metop-B, SAC-C and TerraSAR-X missions, combining them into one gridded dataset for the years 2007-2016. Our approach to extract RWP anomalies and their envelope uses Fourier and Hilbert transforms over longitude without pre- or post-processing the data. Our study is purely based on observations, only using ERA-Interim winds to provide information about the background wind regimes.

The RWP structures that we obtain in the UTLS agree well with theory and earlier studies, in terms of coherent phase or group propagation, zonal scale and distribution over latitudes. Furthermore, we show that RWP pressure anomalies maximize around the tropopause, while RWP temperature anomalies maximize right above the tropopause height with a contrasting minimum right below. RWP activity follows the zonal-mean tropopause during all seasons.

RWP anomalies in the lower stratosphere are dynamically coupled to the upper troposphere. They are part of the same system with a quasi-barotropic structure across the UTLS. RWP activity often reaches up to $20 \mathrm{~km}$ height and occasionally higher, defying the Charney-Drazin criterion. We note enhanced amplitude and upward propagation of RWP activity during sudden stratospheric warmings.

We provide observational support for improvements in RWP diagnostics and wave trend analysis in models and re-
\end{abstract}

analyses. Wave quantities follow the tropopause, and diagnosing them on fixed pressure levels (which the tropopause does not follow) can lead to aliasing. Our novel approach analyzing GNSS-RO pressure anomalies provides wave signals with better continuity and coherence across the UTLS and the stratosphere, compared to temperature anomalies. Thus, RWP vertical propagation is much easier to analyze with pressure data.

\section{Introduction}

Rossby wave packets (RWPs) are transient and zonally confined undulations of the extratropical westerly flow. They can be seen as an organized succession of troughs and ridges with sub-planetary zonal scale and a characteristic timescale from days to a couple of weeks (Wirth et al., 2018). The dispersive nature and downstream development of RWPs has long been noted (Rossby, 1945; Hovmöller, 1949) due to their faster group speed than phase speed (Andrews et al., 1987). RWPs propagate along "waveguides", narrow bands of sharp isentropic potential vorticity (PV) gradients (Hoskins et al., 1985; Shapiro and Keyser, 1990; Martius et al., 2010), and are fueled mainly by baroclinic energy generation (e.g., Chang, 2001), thereby also being referred to as baroclinic waves. RWPs are steered by the polar front jet, reinforcing it via wave-mean flow interaction (Hoskins et al., 1983), and they represent transient states of the storm tracks, regions with frequent cyclone occurrence (Hoskins and Valdes, 1990; Chang et al., 2002).

The horizontal scale of RWPs varies seamlessly between medium (wavenumbers w4-7) and synoptic scale ( $\mathrm{w}>7$ ); 
therefore an intermediate range of w4-15 or similar is generally used to study RWPs (e.g., Zimin et al., 2003; Glatt and Wirth, 2014; Wolf and Wirth, 2017; Quinting and Vitart, 2019). The extent in longitude of RWPs, or the size of the envelope that modulates the wave's amplitude, also shows high variability: in the Southern Hemisphere ( $\mathrm{SH})$ a more global scale is observed, while in the Northern Hemisphere (NH) the waves tend to be more localized, due to the extent and zonality of the waveguide (e.g., Lau, 1979; Randel and Stanford, 1985). Nevertheless, either behavior can occur in both hemispheres at specific times, and even when RWPs reach a global scale, the amplitude of the wave is rarely constant along all longitudes. Global or local wave modes show no apparent differences in their dynamics (Randel and Stanford, 1985). For consistency we will use the term RWP throughout the paper to refer to wave activity, since it simultaneously covers the medium and synoptic scales.

RWPs are the main driver of extratropical weather and climate, also at larger spatial and timescales. Variations in the zonal-mean westerlies of the order of 2-3 weeks were found to originate from energy conversion between zonalmean flow and medium-scale waves in the SH (Webster and Keller, 1975; Randel and Stanford, 1985). Eddy feedbacks are responsible for the increased timescale of annular modes in the extratropical winter troposphere which are coupled with the stratospheric polar vortex behavior (Lorenz and Hartmann, 2001, 2003), making RWPs an important contributor to stratosphere-troposphere coupling (Kidston et al., 2015).

The theory for lower-upper level PV anomaly coamplification (Hoskins et al., 1985), idealized life cycles (Gall, 1976; Simmons and Hoskins, 1978; Thorncroft et al., 1993), and conceptual and case studies (Shapiro and Keyser, 1990) have all highlighted the importance of tropopause processes in the formation and evolution of baroclinic waves for decades, but research has only recently started to focus on the role of the stratosphere. Williams and Colucci (2010) showed that RWP properties in the upper troposphere depended on lower-stratospheric conditions, namely the strength of the polar vortex. New case studies about extreme cyclones are pointing to a significant role of stratospheric conditions in their development (Odell et al., 2013; Tao et al., 2017a, b), and a more general study about severe European windstorms found that in 20 of 60 cases the stratospheric contribution during their deepening phases was over $10 \%$ (Pirret et al., 2017). Nevertheless, despite their interaction with the stratosphere, it is generally assumed that RWPs cannot propagate upward due to the typical wind regimes in the stratosphere (Charney and Drazin, 1961).

There are several issues concerning RWP representation in forecast models. Atmospheric models present a general bias in their simulated waveguides, which are not as sharp as observed (Gray et al., 2014; Giannakaki and Martius, 2016). The horizontal and vertical structures of forecast errors tend to spread together with the RWP's envelope (Dirren et al.,
2003; Hakim, 2005; Sellwood et al., 2008; Zheng et al., 2013), and near-tropopause dynamics in particular are the ones dominating error growth (Baumgart et al., 2018). Apart from baroclinic energy generation, other important processes that affect PV distribution in RWPs near the tropopause (and thereby forecast error growth) are latent-heat release and longwave radiative cooling (e.g., Martínez-Alvarado et al., 2016; Teubler and Riemer, 2016). The organized structure of RWPs and their large traveling distances, compared to individual troughs and ridges or cyclones and anticyclones, offers the opportunity of improving and extending the range of weather forecasts with a better understanding and modeling of their dynamics (Grazzini and Vitart, 2015).

Climate change can have opposing influences on the storm tracks (Shaw et al., 2016) or the polar front jet (Hall et al., 2015) position. As an example, Arctic amplification will decrease the lower troposphere's meridional temperature gradient and baroclinicity, tending to shift the storm track equatorward, while stratospheric radiative cooling by $\mathrm{CO}_{2}$ will increase near-tropopause baroclinicity towards the pole, counterbalancing the shift in the lower troposphere. This is one reason why climate projections show sensitivity to the inclusion of the stratosphere in models (Scaife et al., 2012), and RWP dynamics play a central role in establishing where the projected storm tracks and polar front jets will shift to. Models used for climate projections, e.g., the Coupled Model Intercomparison Project Phase 5 (CMIP5), are known to have biases in their storm tracks (Chang et al., 2012; Hall et al., 2015).

Recognizing the importance of RWPs for weather forecasting, climate change projections and stratospheretroposphere interactions, the scientific community would greatly benefit from increased observational knowledge of RWP structures and behavior across the upper troposphere and lower stratosphere (UTLS), which is the main goal of our study in an area lacking research thus far. The recent availability of Global Navigation Satellite System radio occultation (GNSS-RO) measurements (e.g., Kursinski et al., 1997; Anthes, 2011) enables the study of RWP temperature and pressure anomalies with global coverage across the UTLS at unprecedented high vertical resolution. The exclusive use of 1 decade of GNSS-RO observations (2007-2016) will avoid any limitation from reanalysis or model output in the form of biases or lack of vertical resolution within the UTLS. The present paper intends to describe general and purely observational RWP properties across the UTLS.

GNSS-RO observations have been used to study atmospheric waves in many recent studies. In the equatorial regions, Kelvin waves have been studied the most so far (e.g., Randel and $\mathrm{Wu}, 2005$; Flannaghan and Fueglistaler, 2013; Scherllin-Pirscher et al., 2017), with Zeng et al. (2012) and Kim and Son (2012) also studying the Madden-Julian oscillation (MJO; Madden and Julian, 1994). Alexander et al. (2008) and Pilch Kedzierski et al. (2016a) had a wider approach and considered all equatorial wave types. In the ex- 
tratropics, studies using GNSS-RO measurements have concentrated mainly on the extraction of gravity wave parameters (e.g., Tsuda et al., 2000; Wang and Alexander, 2010; Kohma and Sato, 2011; Tsuda, 2014; Schmidt et al., 2016). Madhavi et al. (2015) studied the $2 \mathrm{~d}$ wave in the upper stratosphere and lower mesosphere in both the $\mathrm{NH}$ and $\mathrm{SH}$ extratropics. Alexander and Shepherd (2010) studied planetary wave activity in the Arctic and Antarctic lower and mid-stratosphere, while Shepherd and Tsuda (2008) studied planetary waves in the $\mathrm{SH}$ polar summer at $30 \mathrm{~km}$ height. Pilch Kedzierski et al. (2017) used GNSS-RO to study extratropical tropopause modulation by waves, although only separating wave activity by timescale and propagation direction, without describing wave properties. Our study is a first attempt to describe RWP properties in the extratropics with the use of GNSS-RO.

This paper is organized as follows: Sect. 2 will introduce the GNSS-RO missions used for our study and the analysis methods applied, Sect. 3 will present how the zonally averaged RWP activity is distributed in the UTLS globally, and Sect. 4 will concentrate on RWP zonal and vertical structures. Both Sects. 3 and 4 will start with case studies to introduce how RWPs evolve across the UTLS, moving on to climatological statistics with a focus on general and common RWP properties in the extratropics of both hemispheres. Section 5 will discuss some implications of our results, and Sect. 6 will summarize our main findings.

\section{Data and methods}

\subsection{Datasets}

We make use of GNSS-RO measurements from the following satellite missions: CHAMP (Wickert et al., 2001), COSMIC (Anthes et al., 2008), GRACE (Beyerle et al., 2005), MetopA (von Engeln et al., 2011), the successive Metop-B, SAC-C (Hajj et al., 2004) and TerraSAR-X (Beyerle et al., 2011). All data are reprocessed or post-processed occultation profiles with moisture information ("wetPrf" product) from the COSMIC Data Analysis and Archive Center (CDAAC, https: //cdaac-www.cosmic.ucar.edu/cdaac/products.html, last access: 15 July 2020), for the years 2007-2016. GNSS-RO profiles of temperature and pressure are provided interpolated on a $100 \mathrm{~m}$ vertical grid between the surface and $40 \mathrm{~km}$ height, although their effective physical resolution varies from $\sim 1 \mathrm{~km}$ in regions of constant stratification down to the order of $100 \mathrm{~m}$ where stratification gradients occur, such as the tropopause or the top of the boundary layer (Kursinski et al., 1997; Gorbunov et al., 2004).

The wetPrf product is the best suited for our study for several reasons. In regions where the atmosphere is dry (roughly above $10 \mathrm{~km}$ height), the wetPrf and the dry temperature "atmPrf" profiles basically coincide (Alexander et al., 2014; Danzer et al., 2014). At lower levels, water vapor is increas- ingly influential and dry temperatures get colder than the real temperature (e.g., at $8 \mathrm{~km}$ height in the extratropics, the difference is already of the order of $0.5-2 \mathrm{~K}$ and increasing downwards in Danzer et al., 2014). Our wave analyses start at $6 \mathrm{~km}$ height, and we note that the extratropical tropopause can vary between 7 and $12 \mathrm{~km}$ height over one latitude band on the same day (see Sect. 4.1 and 4.2). Water vapor content within the RWP's troughs and ridges can be very different at a given vertical level and its effect on the GNSSRO profiles, especially within the lower part of the UTLS, cannot be neglected if reliable wave amplitudes are desired. To account for water vapor effects on the retrieved GNSSRO profile, a background state (typically analyses) is assimilated using one-dimensional variational (1D-Var) technique (Healy and Eyre, 2000; Poli et al., 2002). Although this procedure combines the GNSS-RO measurement with the "firstguess" background, it has been shown that between 9 and $22 \mathrm{~km}$ height the GNSS-RO measurement dominates the retrieved vertical profile (Healy and Eyre, 2000), whereas at lower levels there are temperature improvements from the background towards radiosonde measurements where available (Poli et al., 2002).

Different studies have shown the consistency, mission independence and good precision among different GNSS-RO satellite missions as well as compared to radiosondes (Hajj et al., 2004; Wickert et al., 2009; Schreiner et al., 2011; Anthes et al., 2008; Ho et al., 2017). The advantage of GNSSRO profiles over radiosondes relies on their global coverage, weather independence and higher sampling density. The time period of our analysis spans 2007-2016, and thanks to the simultaneous use of several missions, the total number of GNSS-RO profiles is fairly stable with around 2500 profiles per day.

All GNSS-RO profiles undergo an in-depth quality check (see Sect. 2.2) to eliminate erroneous or unphysical profiles, which, if present often enough, can trigger false signals when applying space-time filters. After merging all GNSSRO data into one gridded dataset (see Sect. 2.3), we find no discontinuities or artifacts arising from the presence of GNSS-RO from different missions, due to the self-calibration and consistency of each satellite instrument and the post(re)processing at the same center (CDAAC). The RWP temperature anomalies we find after filtering our gridded dataset (see Sect. 2.4) are 1-2 orders of magnitude higher than the spread of large-scale and long-term temperature differences across different GNSS-RO processing centers, depending on the height range (Steiner et al., 2020).

The GNSS-RO measurements are complemented with zonal-mean zonal wind data from the ERA-Interim reanalysis (Dee et al., 2011) for the same period 2007-2016, in order to provide a context about background wind regimes. The programming language " $R$ " (R Core Team, 2015) is used to perform the quality control (Sect. 2.2), gridding (Sect. 2.3) and wave analysis (Sect. 2.4) from the GNSS-RO data. 


\subsection{Quality control for GNSS-RO profiles}

The quality control for the GNSS-RO measurements from all satellite missions is performed in two steps.

1. The first step is intended as a general screening for profiles whose temperatures or tropopause stability fall outside global climatological values. Temperature profiles with values $<-100$ or $>50^{\circ} \mathrm{C}$ are excluded, as are those with $T<-90^{\circ} \mathrm{C}$ above $35 \mathrm{~km}$ height, since the coldest values within the winter polar vortex occur well below $35 \mathrm{~km}$. The tropopause height $\left(\mathrm{TP}_{z}\right)$ is defined following the World Meteorological Organization lapse-rate criterion (WMO, 1957). Profiles where the tropopause cannot be found, or those with tropopauses that are unreasonably warm $\left(>-45^{\circ} \mathrm{C}\right)$, are excluded. This is close to the mean tropopause temperature in polar summer $\left(-50^{\circ} \mathrm{C}\right)$, but we do not find discontinuities in the time availability of GNSS-RO profiles arising from this criterion. Static stability is calculated as the Brunt-Väisälä frequency squared, $N^{2}=$ $(g / \Theta) \cdot(\partial \Theta / \partial z)$, where $g$ is the gravitational acceleration, $\Theta$ the potential temperature and $\partial z$ its vertical derivative. The $N^{2}$ maximum above $\mathrm{TP}_{z}$ (up to $3 \mathrm{~km}$ ) is computed, and profiles with tropospheric values $\left(N^{2}<\right.$ $\left.3 \times 10^{-4} \mathrm{~s}^{-2}\right)$ or values that are too high $\left(N^{2}>100 \times\right.$ $10^{-4} \mathrm{~s}^{-2}$ ) are excluded. These conditions are based on Son et al. (2011) for $\mathrm{TP}_{z}$ temperature climatologies from GNSS-RO measurements and Pilch Kedzierski et al. (2016a) for maximum $N^{2}$ climatologies, whose highest values are found in the tropics. From the initial 10053153 profiles available for 2007-2016 from all GNSS-RO satellite missions, this first step keeps $9369092(93.2 \%)$ of them.

2. After step 1 there remains a significant number of GNSS-RO profiles whose stratospheric temperatures markedly stand out when compared to nearby occultations in space and time, although passing the climatological criteria. On a global $5^{\circ}$ by $5^{\circ}$ longitude-latitude grid, for each day throughout 2007-2016 and at every grid point, GNSS-RO profiles within $\pm 3 \mathrm{~d}, \pm 10^{\circ}$ longitude and $\pm 5^{\circ}$ latitude are selected. A distribution of their $30 \mathrm{~km}$ temperature is computed, and a mean temperature profile is calculated from those GNSSRO profiles that fall between the 0.2 and 0.8 quantiles, which avoids the influence of possibly erroneous profiles. The integrated squared temperature difference from the mean profile between 20 and $40 \mathrm{~km}$ height is calculated for each occultation: $A_{i}=\sum_{20 \mathrm{~km}}^{40 \mathrm{~km}}\left(T_{i}^{\prime}\right)^{2}$. The 0.5 quantile of $\mathrm{A}$ represents the half of the selected GNSS-RO profiles that are closer to the mean profile. The profiles with $A_{i}$ exceeding 20 times the 0.5 quantile (that fall far out of the distribution) are excluded. Step 2 eliminates GNSS-RO profiles with unrealistic stratospheric temperatures for their season and location, and even in extreme situations with stark temperature contrasts such as sudden stratospheric warmings this criterion is not met.

After the quality control is carried out, 9215804 profiles (91.7\% of the initial GNSS-RO dataset) are used.

\subsection{Gridding}

The method to grid GNSS-RO profiles in our study is a refinement of Pilch Kedzierski et al. (2017) and Pilch Kedzierski et al. (2016a), with better horizontal resolution and with ground-based instead of tropopause-based averaging. Both were developed after the method by Randel and Wu (2005). GNSS-RO profiles of temperature and pressure for the years 2007-2016 are gridded daily on a $5^{\circ}$ by $5^{\circ}$ longitude-latitude grid, from $85^{\circ} \mathrm{S}$ to $85^{\circ} \mathrm{N}$. The height range analyzed is between 6 and $40 \mathrm{~km}$, although because the focus of this paper is the UTLS region, most of the presented results will use a lower lid. The amount of GNSS-RO profiles that penetrate deeper than $6 \mathrm{~km}$ diminishes at lower altitudes. Having fewer data available to grid forces more interpolation and less reliability of filtered signals at lower levels. The GNSS-RO profiles that fall within the grid point area are averaged as follows:

$\begin{aligned} T_{\text {grid }}(\lambda, \phi, z, t) & =\sum_{i} w_{i} T_{i}(\lambda, \phi, z, t) / \sum_{i} w_{i}, \\ P_{\text {grid }}(\lambda, \phi, z, t) & =\sum_{i} w_{i} P_{i}(\lambda, \phi, z, t) / \sum_{i} w_{i},\end{aligned}$

where $\lambda$ is longitude, $\phi$ is latitude, $z$ is height and $t$ is time. The weight $w_{i}$ is a Gaussian function determined by each GNSS-RO profile's distance from the grid center:

$w_{i}=\exp \left(-\left[\left(D_{x} / 2.5\right)^{2}+\left(D_{y} / 2.5\right)^{2}+\left(D_{t} / 12\right)^{2}\right]\right)$,

with $D_{x}$ and $D_{y}$ as the distances in degrees longitude and degrees latitude from the grid's center and $D_{t}$ as the time distance in hours from 12:00 UTC, divided by the grid's half size in all dimensions: $2.5^{\circ}$ longitude, $2.5^{\circ}$ latitude and $12 \mathrm{~h}$.

Typically 2-3 GNSS-RO profiles of the same day are selected within the grid's area for averaging, with the following exceptions. In a $28 \%$ of grid points the allowed distance to search for GNSS-RO profiles needs to be expanded to $\pm 5^{\circ}$ longitude and $\pm 3.5^{\circ}$ latitude in order to avoid gaps (in $9 \%$ of cases it expands further to $\pm 10^{\circ}$ longitude and $\pm 5^{\circ}$ latitude), without changing the weighting function $w_{i}$ in any case. The remaining $1 \%$ of gaps are filled by averaging adjacent grid points ( \pm 1 in longitude, then \pm 1 in time). The exception's percentages presented here belong to the Northern Hemisphere from $15^{\circ}$ to $85^{\circ}$ latitude and are nearly identical in the Southern Hemisphere. The gridded tropopause height $\operatorname{TP}_{z}(\lambda, \phi, t)$ is calculated with the same weighting of all profiles' tropopauses.

Overall the real resolution of the gridded dataset is slightly coarser than $5^{\circ}$ longitude-latitude and may be viewed as an 
interpolation to a certain degree. Nonetheless this setting resolves the horizontal scale of the RWPs analyzed in our study very well (see Sects. 3 and 4).

\subsection{Wave analysis}

Given that the main goal of this paper is to describe properties of RWPs in the UTLS in a very general manner, we intend to keep the wave analysis as simple as possible. In most analyses a fast Fourier transform (FFT) in longitude is used to extract the wave anomalies from the gridded GNSS-RO temperature and pressure data, either for individual wavenumbers (Sect. 3) or using an intermediate range typical of RWPs (Sect. 4). For RWP envelope reconstruction, the Hilbert transform (Zimin et al., 2003) is applied to RWP pressure anomalies without any further pre- or postprocessing of the RWP anomalies or their envelope (Sect. 4). A couple of exceptions that add a degree of complexity to the analysis are disclosed below.

FFT in longitude and time. To avoid including stationary waves when showing the climatological distribution of RWP activity over latitudes, we use a two-dimensional FFT in longitude and time (Schreck, 2009) to keep the transient components of each wavenumber (eastward-propagating, 2-20 d periods). This is only used for Figs. 3 and 4 in Sect. 3.2.

Vertical scale analysis. RWP anomalies are extracted by taking zonal wavenumbers 4-8 with an FFT in the longitude dimension, obtaining daily longitude-height snapshots. In a second step, at every longitude grid an additional FFT is performed in the vertical dimension on the profile of RWP anomalies between 6 and $36 \mathrm{~km}$ height, in order to obtain the power spectrum of the different vertical wavelengths. The power spectra are then averaged for midlatitudes in Fig. 10 in Sect. 4.2.

Zonality of RWP envelopes. An FFT in longitude is used to obtain RWP anomalies (w4-8), on which the Hilbert transform is applied to reconstruct the RWP envelope. In a third step, another FFT in longitude is applied to the reconstructed envelope to obtain its zonal wavenumber power spectrum. The average wavenumber spectra of RWP envelopes at midlatitudes are shown in Fig. 12 in Sect. 4.2.

Equivalence of RWP pressure anomalies to geostrophic meridional winds. The RWP pressure anomalies in our study are defined relative to the zonal-mean pressure at each level: let us name it $P^{\prime}$ rel $=P^{\prime} / \bar{P}$. This notation is very convenient, since $P^{\prime}$ rel is proportional to geostrophic meridional winds as explained next.

Under geostrophic balance, $f v_{g}=\frac{1}{\rho} \cdot \frac{\delta P}{\delta x}$, with $f$ the Coriolis parameter, $v_{\mathrm{g}}$ geostrophic meridional wind, $\rho$ air density, $P$ pressure and $\delta / \delta x$ its partial derivative in the longitude dimension. Knowing $\rho=P / R T$ where $R$ is the specific gas constant for air and $T$ absolute temperature,

$v_{\mathrm{g}}=\frac{R T}{f} \cdot \frac{\delta P}{P \delta x} \propto \frac{\delta P_{\mathrm{rel}}^{\prime}}{\delta x}$.
Note that both $T$ and $P$ in Eq. (4) are in absolute terms, with zonal variations being relatively small and therefore influencing $v_{\mathrm{g}}$ much less than the $\frac{\delta P}{\delta x}$ term. It follows that wave amplitudes of $v_{\mathrm{g}}$ and $P^{\prime}$ rel should remain proportional over a given height range. Our results throughout Sects. 3 and 4 indicate that $P^{\prime}$ rel decreases away from the tropopause, which agrees with $v_{\mathrm{g}}$ resulting from jet stream undulations whose wind maxima are located nearby.

\section{Distribution of wave activity in the extratropical UTLS}

Section 3 concentrates on the analysis of wave activity as the amplitude of individual harmonics, which only allows us to study RWPs from a zonally integrated perspective. Section 3.1 will introduce examples of wave activity behavior from gridded temperature and pressure GNSS-RO data for 1 year and one latitude band: July 2008 till June 2009 at $50^{\circ}$ N. Section 3.2 will generalize those results with climatological statistics for the 2007-2016 period throughout the whole extratropics. Results are presented this way so the reader gets an overall impression of where wave activity is located as well as how it evolves on a day-to-day basis. Section 4 will analyze RWP zonal structures and their horizontal propagation.

\subsection{Time-height section examples}

We begin by analyzing RWP activity in time-height sections. Figure 1 shows the evolution of the amplitudes of wavenumbers 4 to 8 during 2008-2009 at 50 $\mathrm{N}$, filtered from gridded GNSS-RO temperature fields. The $50^{\circ} \mathrm{N}$ latitude represents the one with most wave activity in the $\mathrm{NH}$ extratropics. The highest amplitudes (orange and red shading) are found directly above the zonal-mean lapse-rate tropopause (magenta line) and in the upper troposphere, with a stark minimum in temperature signals in between (blue shading). This vertical distribution of wave activity in terms of temperature tightly follows the zonal-mean tropopause over time. Wavenumbers 4 and 5 reach amplitudes of 6-7 K, with higher wavenumbers showing lower amplitudes: 3-4 K for w8.

The activity of all wavenumbers in general seems to be confined within westerly winds (solid grey lines): very little penetrates into the summer easterlies (dashed white lines). Interestingly, stratospheric zonal-mean zonal winds of 10 $20 \mathrm{~m} \mathrm{~s}^{-1}$ are no impediment for wave activity to propagate beyond $20 \mathrm{~km}$ height, which can be seen most clearly for wavenumbers 4 to 6 : they show amplitudes in excess of $4 \mathrm{~K}$ around $20-26 \mathrm{~km}$ height before and during a sudden stratospheric warming (SSW) with a central date on 24 January 2009, whose wind anomalies at $50^{\circ} \mathrm{N}$ can be seen until April in the lower stratosphere, with a patch of easterly winds (thick white lines) starting in February 2009 at $26 \mathrm{~km}$. 

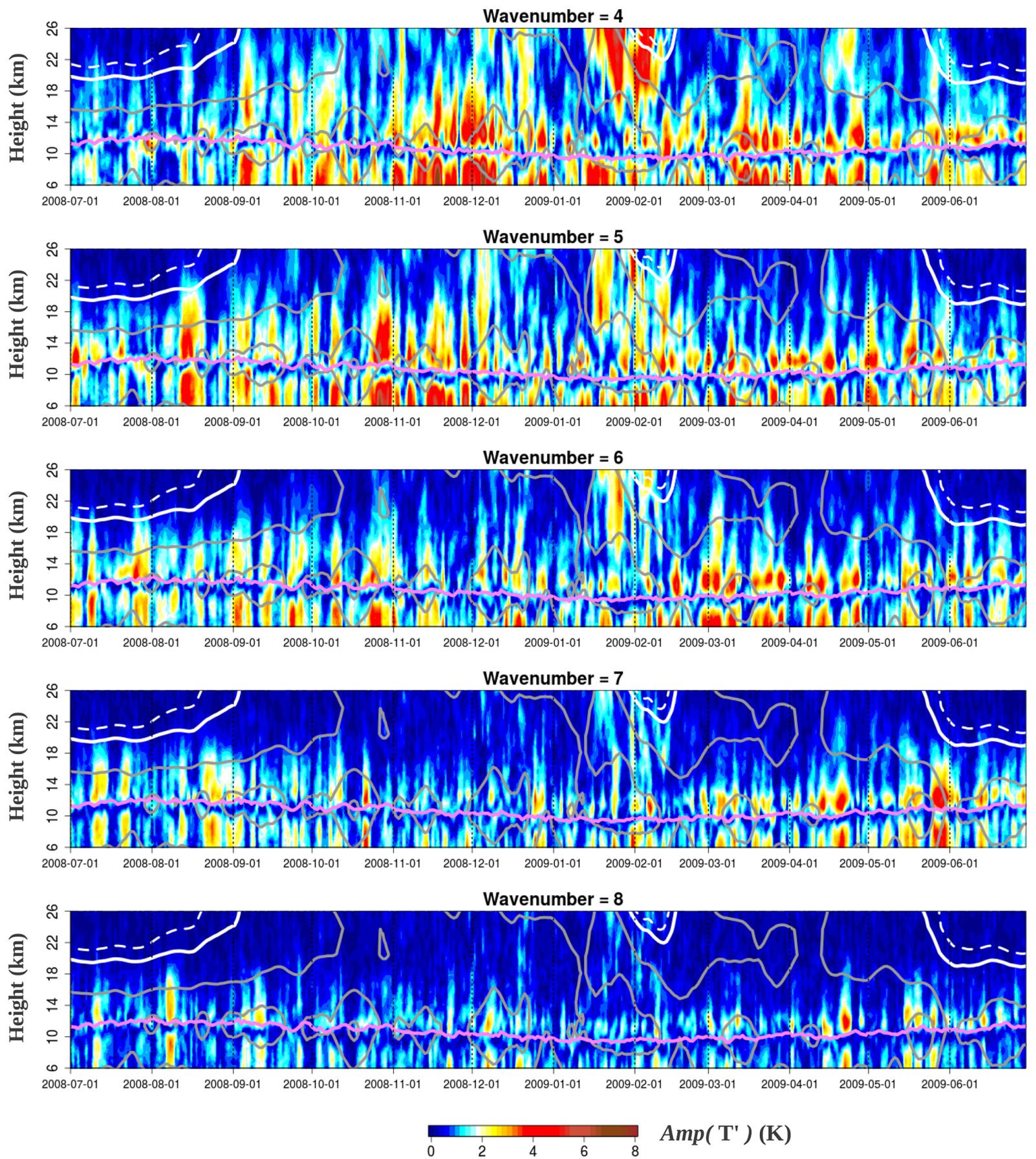

Figure 1. Time-height sections of wave activity for wavenumbers $4-8$ at $50^{\circ} \mathrm{N}$, in terms of the amplitude of the individual harmonics in temperature (colors). Magenta line denotes the zonal-mean $\mathrm{TP}_{z}$. Grey solid lines denote westerly zonal winds, with $10 \mathrm{~ms} \mathrm{~s}^{-1}$ separation. Thick white solid and dashed lines denote 0 and $-3 \mathrm{~ms}^{-1}$, respectively. To improve visibility, the ERA-Interim zonal-mean zonal wind is displayed with a running mean of $\pm 15 \mathrm{~d}$.

All wavenumbers show temperature signals of $2-3 \mathrm{~K}$ (yellow shading) reaching $18-20 \mathrm{~km}$ height very often. The observed GNSS-RO temperature signals of wavenumbers $4-8$ reaching that high into the stratosphere in Fig. 1 is in con- tradiction with the Charney-Drazin criterion (Charney and Drazin, 1961).

In Fig. 1 it may also be noticed that the temperature signals in the lower stratosphere (LS) and the upper troposphere (UT) tend to amplify and dissipate simultaneously, 
apart from showing very similar amplitudes. For wavenumbers 6 and higher this happens throughout the whole year; for w5 and w4 it happens less during the summer months. The vertical propagation of the temperature signals is difficult to discern in Fig. 1. There are hints of rapid upward propagation in the stratosphere which are better visible in the analysis of pressure signals, which will be discussed later in this section.

It should pointed out that the wave signals shown in Fig. 1 mostly belong to traveling waves: if the same figure is done with signals filtered also in time (eastward-propagating with 2-20 d periods), it looks very similar (not shown). We proceed to repeat the analysis from Fig. 1 on GNSS-RO gridded pressure fields. The filtered pressure anomalies are displayed relative to the mean pressure of their vertical level (in percentage units) to make the comparison between tropospheric and stratospheric signals fairer, given the exponential decrease in pressure with height. Also, this notation is proportional to wave amplitude in terms of geostrophic meridional winds as explained in Sect. 2.4.

Figure 2 shows time-height sections of the wave pressure amplitudes (in percent of the zonal-mean pressure) of wavenumbers 4 to 8 , also for $50^{\circ} \mathrm{N}$ and 2008-2009. The same bursts of wave activity can be observed as in Fig. 1, but the most striking difference is that the pressure anomalies in Fig. 2 maximize around the tropopause height, following it constantly throughout the year for all studied wavenumbers. Wavenumbers 4 and 5 reach amplitudes of 3\%-4\% (dark red and brown shading), diminishing towards higher wavenumbers: $\sim 1.5 \%$ for $\mathrm{w} 8$.

In Fig. 2 the wave pressure anomalies appear to form around the tropopause height and radiate outward vertically, diminishing their amplitude away from the tropopause. Wavenumbers 4 and 5 (and w6 in smaller amounts) show frequent upward propagation in the winter stratosphere well beyond $20 \mathrm{~km}$ height. Although generally decaying with height, in some cases higher wave amplitudes (red shading) are retained even at $26 \mathrm{~km}$ height: w4 at the beginning of December 2008 and mid-January 2009 or w5 in January 2009. Wave activity in terms of pressure of w7 and w8 tends to be confined closer to the tropopause, with very little activity reaching beyond $18 \mathrm{~km}$ height in Fig. 2. Compared to Fig. 1, the wave pressure anomalies from Fig. 2 show a much betterdefined continuity in their upward propagation in the stratosphere, making them much easier to track in time-height sections. For example, w4 and w5 temperature anomalies in January 2009 in Fig. 1 show no attachment between the lower and middle stratosphere (red patches are separated by blue regions of very low temperature amplitudes). Meanwhile, the corresponding pressure anomalies in Fig. 2 show clear continuity and upward propagation from the lower to the middle stratosphere. This can be discerned in the w4 bursts in the beginning of December 2008, mid-March 2009 and midApril 2009, with the red and yellow tracks being tilted upward in the positive time direction in Fig. 2. In January 2009, the vertical propagation of several w5 bursts up to $26 \mathrm{~km}$ height is observed coinciding with the 2009 SSW. In this case, $\mathrm{w} 4$ vertical propagation seems to be very rapid in January 2009 (the red track appears quasi-vertical), maintaining very high wave amplitude throughout the whole lower and middle stratosphere. Interestingly w4, w5 and w6 to some degree show some re-amplification of their pressure anomalies near the zero-wind line (thick solid white line in Fig. 2), which is propagating downward during the 2009 SSW. This should not be interpreted as downward propagation of the RWP pressure anomalies, only that they encounter their critical level for upward propagation there, amplifying before breaking.

Overall the temperature wave structures in Fig. 1 appear much noisier and with more discontinuities compared to the same analysis done with pressure data in Fig. 2, which provides a much cleaner view of wave activity location and propagation. One explanation for this could be that the pressure data show the dynamical structure of the wave which remains together as one system. The thermal structure of the same wave is subject to more variations due to heat fluxes, meridional temperature gradients, energy conversion, etc. which feed the wave but need not be proportional to its dynamical structure locally. To our knowledge, our study is the first to analyze wave activity in terms of pressure with GNSSRO data: only temperature or parameters derived from it have been used to study waves thus far. From the results presented in Fig. 2, the benefits of analyzing pressure GNSS-RO data for wave analysis are noticeable.

From Figs. 1 and 2 it can be concluded that the separate temperature anomalies in the UT and LS and their simultaneous amplification are part of the same pressure system that goes across the UTLS and occasionally propagates far up into the stratosphere, more often for wavenumbers 4 and 5 . The wave pressure anomalies in Fig. 2 that maximize around the zonal-mean tropopause height can be considered equivalent to the upper-level PV anomalies (Hoskins et al., 1985) which then couple with surface circulation. Our results are in agreement with Teubler and Riemer (2016), who showed PV anomalies maximizing around the tropopause height. Since our gridded GNSS-RO dataset includes altitudes of $6 \mathrm{~km}$ and higher, the wave behavior in the lower troposphere cannot be diagnosed to study its coupling with the near-tropopause anomalies, but this will be the subject of future work in combination with other datasets.

Another topic of high interest for future work is the behavior of RWP activity, namely w4 and w5, and the possible influence on SSWs since in Fig. 2 we observe increased propagation into the stratosphere around the time of the 2009 SSW. We see a similar behavior of RWP activity in the 2010 and 2013 SSW cases (see and compare Figs. S1, S2 and S3 in the Supplement). Domeisen et al. (2018) showed important phase speed and amplitude changes of w1 and w2 at the $100 \mathrm{hPa}$ level ( $\sim 16 \mathrm{~km}$ height) preceding the onset of SSWs. The lower stratosphere plays a key role in controlling the downward propagation of SSW anomalies and their coupling 

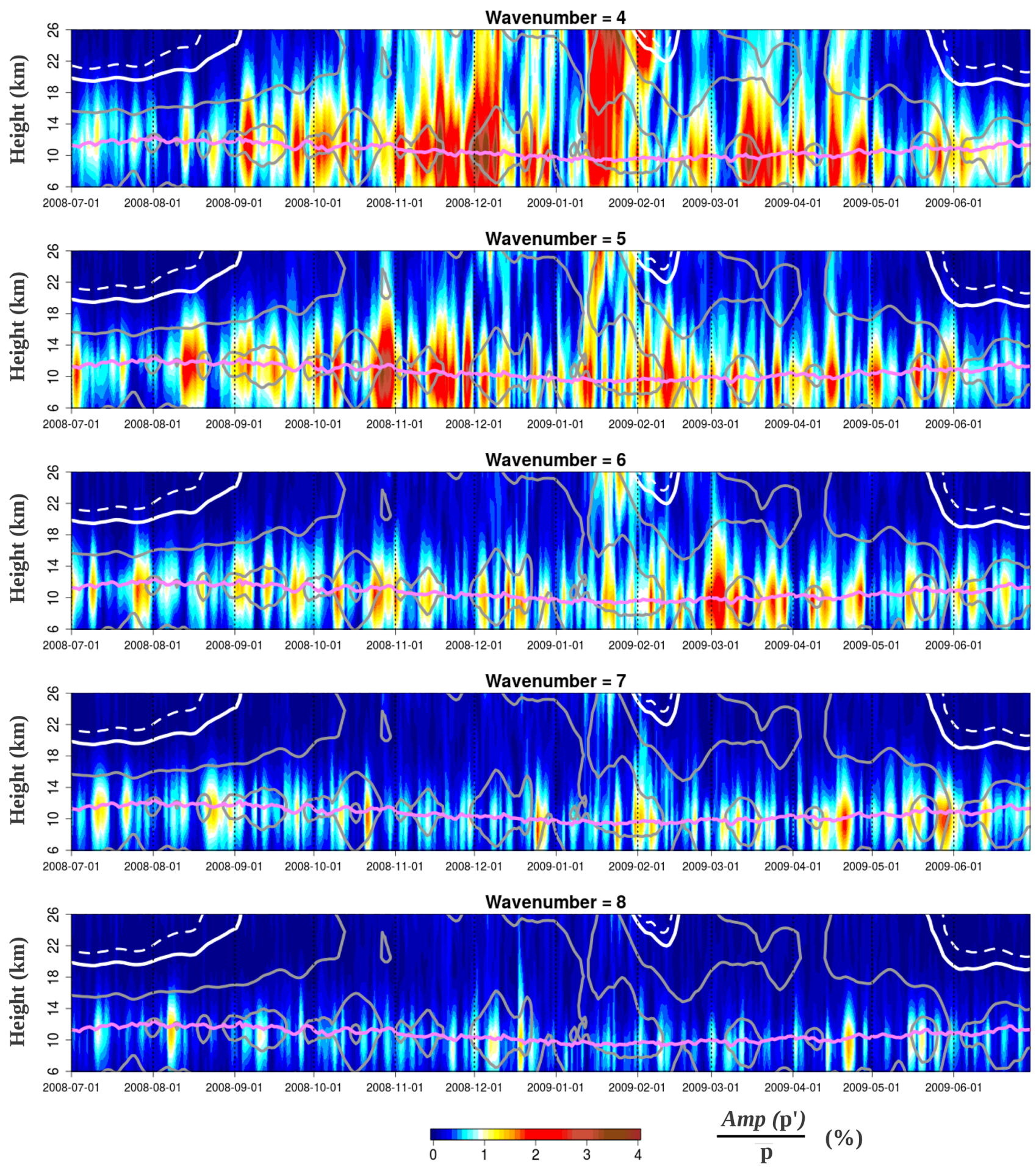

Figure 2. Same as in Fig. 1 but for wave pressure anomalies, relative to the mean pressure of each vertical level.

with the troposphere: Karpechko et al. (2017) showed that northern annular mode (NAM) anomalies at the $150 \mathrm{hPa}$ level define which SSWs affect the troposphere. RWP activity is known to force low-frequency variability of the zonal-mean circulation (Webster and Keller, 1975; Randel and Stanford, 1985)

Given the large amounts of RWP activity we diagnosed in the LS (and stratosphere during SSWs; see Fig. 2), RWP interaction with the LS mean flow through which plane- 
tary waves propagate should be expected: this would affect LS NAM conditions that control SSW downward propagation (Karpechko et al., 2017) and indirectly affect the phase speeds of upward-propagating planetary waves (Domeisen et al., 2018) by Doppler shifting. One may even speculate RWPs could directly affect planetary wave phase speeds and amplitudes in the LS if some wave-wave interaction and energy transfer or positive interference happened between the two. In either case, we state that RWP activity in the LS is potentially relevant for both onset and downward propagation stages of SSWs, opening an interesting field of research.

Figures 1 and 2 have shown an example for 1 year (July 2008 till June 2009) and one latitude band: $50^{\circ}$ N. Section 3.2 will generalize the results shown above, giving climatological statistics for all extratropical latitudes from a decade (2007-2016) of GNSS-RO data.

\subsection{Climatological distribution of wave activity over latitude and height}

How is the wave activity of individual wavenumbers distributed over the whole extratropical latitude range? Taking the zonal-mean tropopause level as a reference, since wave activity in terms of pressure follows it and maximizes there (Fig. 2), we calculate the mean amplitude of each wavenumber's filtered pressure signal. We select the eastward-propagating components with $2-20 \mathrm{~d}$ periods, in order to isolate traveling waves throughout the $\mathrm{NH}$ extratropics. The resulting wave spectrum is shown in Fig. 3a for the whole NH gridded GNSS-RO dataset (2007-2016) and separated into winter and summer climatologies in Fig. $3 \mathrm{~b}$ and c.

The spectrum from Fig. 3a has a similar shape to the spectrum from Wolf and Wirth (2017) (their Fig. 6). Although the parameter used by Wolf and Wirth (2017) is $300 \mathrm{hPa}$ meridional winds and the time filtering is slightly different $(30 \mathrm{~d}$ high-pass), their spectrum also shows the two relative maxima of wave activity: one at midlatitudes around w6 and the other at polar latitudes and lower wavenumbers, similarly to our Fig. 3a. Traveling waves tend to have a similar range of wavelengths at all latitudes, and the spectrum shape results from their resulting zonal wavenumber Fourier composition at the different latitudes.

The wave spectrum for NH winter (Fig. 3b) is more elongated towards lower latitudes, reaching $30^{\circ} \mathrm{N}$. Meanwhile in the NH summer spectrum (Fig. 3c), there is very little wave activity south of $45^{\circ} \mathrm{N}$, which agrees well with the seasonality of the jet stream position. Interestingly, transient wave activity in the Arctic region (poleward of $70^{\circ} \mathrm{N}, \mathrm{w} 1-3$ ) seems to be very active all year round at the zonal-mean tropopause level.

We take the time series of the amplitude of wave temperature anomalies $1.5 \mathrm{~km}$ above and $3 \mathrm{~km}$ below the zonal-mean tropopause at each latitude band and separate wavenumber as a measure of LS and UT thermal wave activity. These heights were chosen to avoid the minimum in wave activity in terms of temperature around and right below the tropopause in Fig. 1. The correlation of the LS and UT time series will indicate the degree of their simultaneous amplification, which is shown in Fig. 3d-f. After seeing Figs. 1 and 2, one would expect a tendency of the upper and lower rows in Fig. 3 to match, but this is not the case: higher values of the measure for simultaneous amplification are shifted towards higher wavenumbers at all seasons. Furthermore, the NH summer (Fig. 3f) shows markedly higher correlations than the $\mathrm{NH}$ winter season (Fig. 3e). The results shown in Fig. 3d-f indicate that the simultaneous amplification of wave temperature signals between the UT and LS is done more towards the synoptic scale and the upper end of the medium scale of the wave spectrum, any season. This is an unexpected finding. One possibility of explaining it would be that the temperature signal requires meridional advection to be formed, and meridional winds have been shown to have a trough-ridge asymmetry (Wolf and Wirth, 2015, 2017) due to their semi-geostrophic nature, with the strongest meridional winds found closer to the trough's center. Therefore, due to non-geostrophic processes, meridional winds and temperature could typically have slightly shorter wavelengths compared to their corresponding pressure anomalies' wavelength. Proving this would require the use of other datasets beyond GNSS-RO; thus this is beyond the scope of our study.

We repeat the same analysis from Fig. 3 with RWP activity latitudinal distribution and the UT-LS simultaneous amplification measure in the Southern Hemisphere (Fig. 4). The wave spectra, as well as the UT-LS simultaneous amplification measure, are qualitatively similar in the NH (Fig. 3) and SH (Fig. 4). The SH shows maximized wave activity at $\mathrm{w} 4$ and $\sim 55^{\circ} \mathrm{S}$ throughout the year, with markedly higher values in terms of mean relative pressure amplitude: SH maxima reach $\sim 1.3 \%$ in Fig. $4 \mathrm{a}-\mathrm{c}$ vs. $\sim 0.7 \%$ in the NH in Fig. 3a-c. SH midlatitudes show increased eastward-propagating wave activity overall, maximized at lower wavenumbers compared to the $\mathrm{NH}$ midlatitudes, in agreement with previous studies (see Randel and Stanford, 1985, and references therein). This interhemispheric difference in the wave spectrum can be explained by the absence of mountains in the SH: a model study by Hayashi and Golder (1983) noted a similar change in the wave spectrum when NH mountains were removed.

The maximum of SH transient wave activity shows little seasonal variation in the latitude where it is located (Fig. 4b and c), although in winter it shows a broader latitudinal extent, indicating larger meridional advection scales. In contrast to the $\mathrm{NH}$, the $\mathrm{SH}$ polar latitudes show very little wave activity during summer months (Fig. 4c). As in the NH, the simultaneous amplification of SH wave temperature signals in the UT and LS is maximized at higher wavenumbers and during the summer months (Fig. 4d-f). The SH distribution of the UT-LS correlation factor, relative to the distribution 


\section{Average pressure amplitudes at zonal-mean tropopause height}

(a) All months

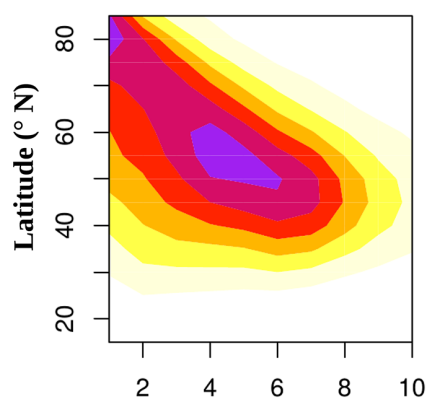

(b) Winter (JFM)

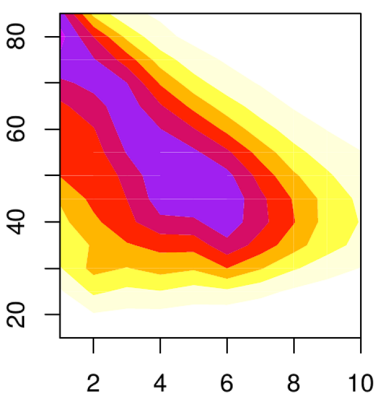

(c) Summer (JAS)

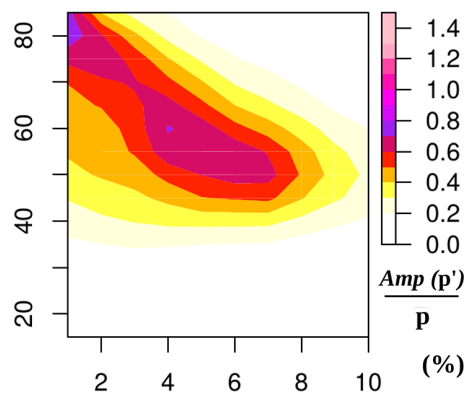

Simultaneous UT and LS temperature signal amplification

(d) All months

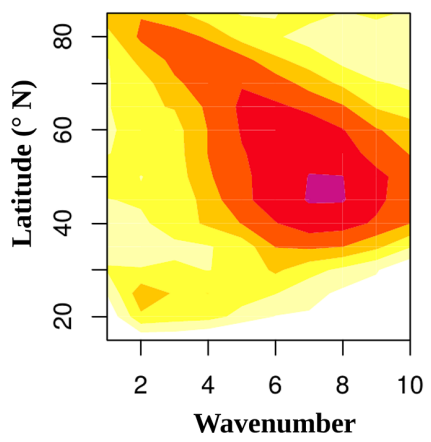

(e) Winter (JFM)

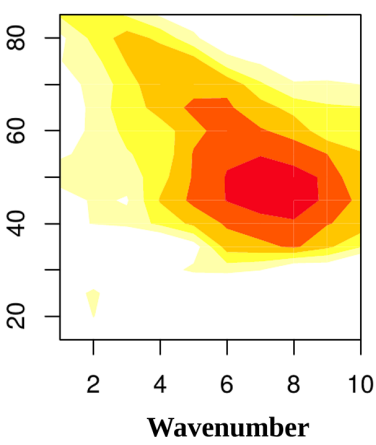

(f) Summer (JAS)

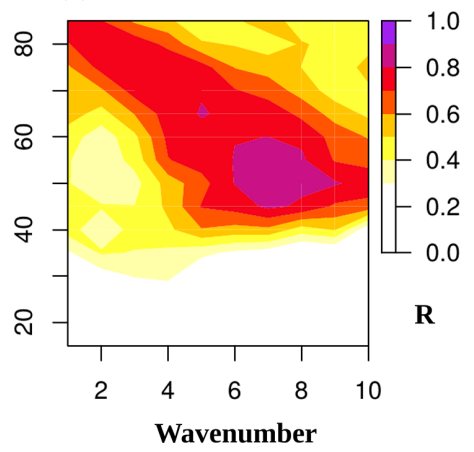

Figure 3. (a-c) Wave activity spectra, as the mean amplitude of the filtered pressure anomalies at the zonal-mean tropopause level for (a) the whole 2007-2016 series, (b) winter and (c) summer months. (d-f) Simultaneous amplification of the LS and UT temperature signals, as the correlation of their amplitude time series for (d) all, (e) winter and (f) summer months.

of transient wave activity, is very similar to the $\mathrm{NH}$ both in magnitude and shape.

We proceed to show a climatology of the vertical distribution of RWP activity in both hemispheres. We noted earlier in Figs. 1 and 2 that the temperature signal had a relative minimum near and right below the zonal-mean tropopause, and a maximum located right above it, while the pressure signal maximized and followed the zonal-mean tropopause. We take the standard deviation (SD) of zonal wavenumbers 48 daily temperature and pressure anomalies and average the daily SD value relative to the zonal-mean tropopause level at both $\mathrm{NH}$ and $\mathrm{SH}$ midlatitudes. We select $\mathrm{w} 8$ as the upper limit because we note very little wave activity at higher wavenumbers from Figs. 3 and 4. The resulting mean vertical profiles of RWP activity are shown in Fig. 5.

The vertical distribution of the RWP temperature signals in Fig. 5a is very similar in both $\mathrm{NH}$ and $\mathrm{SH}$ midlatitudes, with tropospheric and LS relative maxima above $\sim 3 \mathrm{~K}$ and a minimum close to and below the zonal-mean tropopause level of $\sim 1.5 \mathrm{~K}$. The pressure signals in Fig. $5 \mathrm{~b}$ maximize around and below zonal-mean tropopause height, with the SH reaching slightly higher values $(\sim 1.8 \%)$ than the $\mathrm{NH}(\sim 1.5 \%)$, but they are otherwise alike in shape. The higher RWP activ- ity in the SH was already noted comparing the wave spectra from Figs. 3a-c and 4a-c. The climatology in Fig. 5 shows the expected vertical distributions from the $50^{\circ} \mathrm{N}$ examples from Figs. 1 and 2 and shows very little interhemispheric differences. Our climatological profile of RWP temperature signals (w4-8) qualitatively agrees very well with the seasonal averages for individual wavenumbers (w4-7) by Randel and Stanford (1985) (their Fig. 4) and also with the vertical distribution of sensible heat fluxes in Blackmon and White (1982) (their Figs. 4-5). Our climatological profile of RWP pressure signals shows very good agreement with the vertical distribution of momentum fluxes in Blackmon and White (1982) (their Figs. 7-8) as well as the seasonal vertical profiles of w5 geopotential height amplitudes in the SH in Hirooka et al. (1988) (their Fig. 8a). Also, early model experiments predicted RWP activity in terms of geopotential or eddy kinetic energy to maximize around the tropopause (Gall, 1976; Simmons and Hoskins, 1978).

\section{Zonal and vertical structures of RWPs}

A classical RWP example with eastward phase propagation and faster group speed will be presented in Sect. 4.1 with the 
Average pressure amplitudes at zonal-mean tropopause height

(a) All months

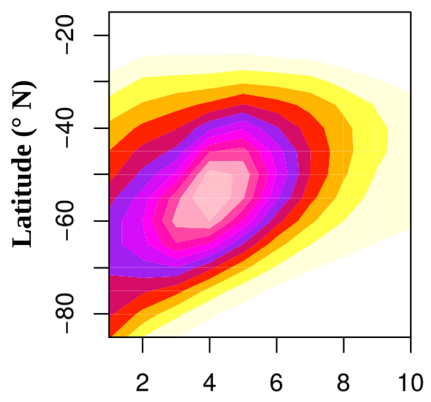

(b) Winter (JAS)

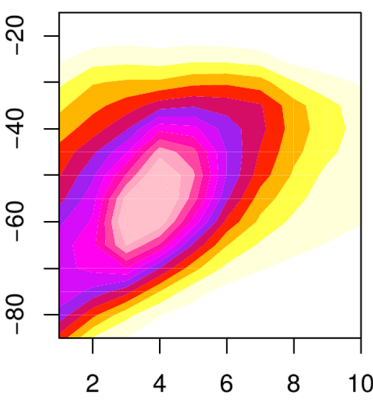

(c) Summer (JFM)

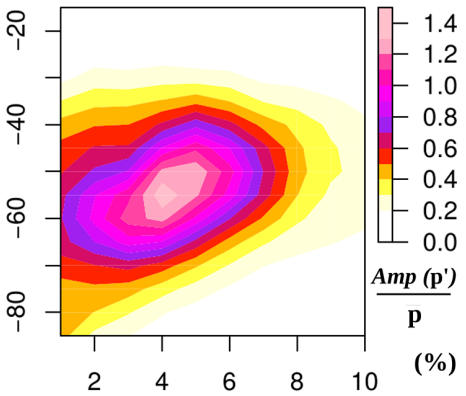

Simultaneous UT and LS temperature signal amplification

(d) All months

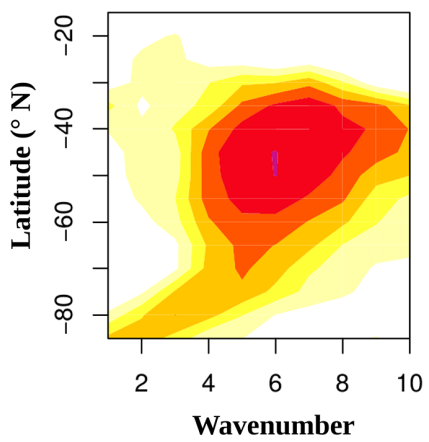

(e) Winter (JAS)

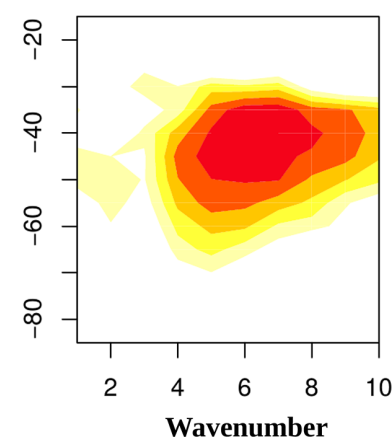

(f) Summer (JFM)

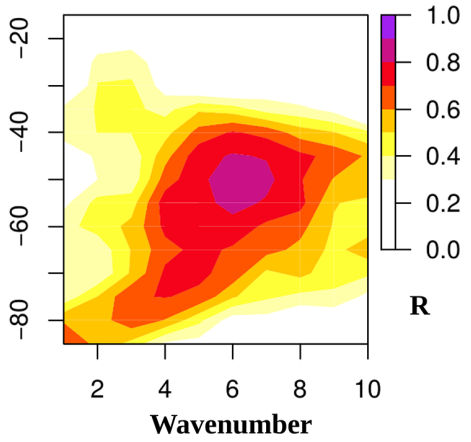

Figure 4. Same as Fig. 3 but for the Southern Hemisphere.

use of Hovmöller diagrams and longitude-height snapshots of RWP anomalies from the gridded GNSS-RO data. Recent studies have pointed out that under specific resonance conditions, RWPs can become stationary and form large-scale teleconnection patterns in midlatitudes, which have been linked to the extreme heat waves of 2003, 2010 and 2018 (Petoukhov et al., 2013; Kornhuber et al., 2017, 2019). To test whether RWP properties in the UTLS are different under these conditions, we will repeat the same analysis from Sect. 4.1 during the Russian heat wave in summer 2010, which will be presented in Sect. 4.2. After these two casestudies, in Sect. 4.3 we will present climatological statistics of the vertical scale of RWP anomalies, the relation of UT and LS zonal temperature structures, and the zonal scale of RWP envelopes for both NH and SH.

\subsection{Classical RWP example}

RWP activity was shown to follow the zonal-mean tropopause in Figs. 1 and 2; therefore we take this level to extract RWP pressure anomalies from the gridded GNSSRO data. This case study was selected to show a classical RWP with eastward phase propagation and a high-amplitude envelope of faster speed, which is the case at the end of February and beginning of March 2009 at $40-60^{\circ} \mathrm{N}$. The zonal structures of RWPs appear from the combination of a range of intermediate wavenumbers that shape their characteristic carrier wave and envelope (see idealized examples in Zimin et al., 2003; Wolf and Wirth, 2015). We select pressure anomalies belonging to wavenumbers 5-8 in order to avoid stationary waves with w1-4 present at the time. The evolution of the RWP pressure anomalies is shown in the Hovmöller diagram in Fig. 6a, and the RWP envelope after applying the Hilbert transform (Zimin et al., 2003) to the w5-8 anomalies is shown in Fig. 6b.

In Fig. $6 \mathrm{~b}$ the formation of an RWP around 27 February 2009 over the Pacific Ocean $\left(\sim 150^{\circ} \mathrm{W}\right)$ can be observed, with the envelope propagating eastward until 7 March 2009 when it covers the Atlantic Ocean $\left(60-0^{\circ} \mathrm{W}\right)$. The eastward phase propagation of the RWP (individual blue troughs and red ridges) in Fig. 6a can be compared to the markedly faster movement of the envelope (green and dark colors) in Fig. 6b. This RWP reaches amplitudes exceeding $4 \%$. By 7 March 2009, a new RWP is forming again over the Pacific Ocean, this one with an even faster envelope and circumnavigating the globe by 12 March 2009, and even reaching global scale for a couple of days although having lower amplitudes than the first RWP, of around $2 \%-3 \%$. It also has to be noted that low-amplitude fluctuations of $\sim 1 \%$ within the w5-8 range are present almost constantly (e.g., light yellow shading in Fig. 6b). 


\section{RWP activity at $40-60^{\circ}$ latitude}

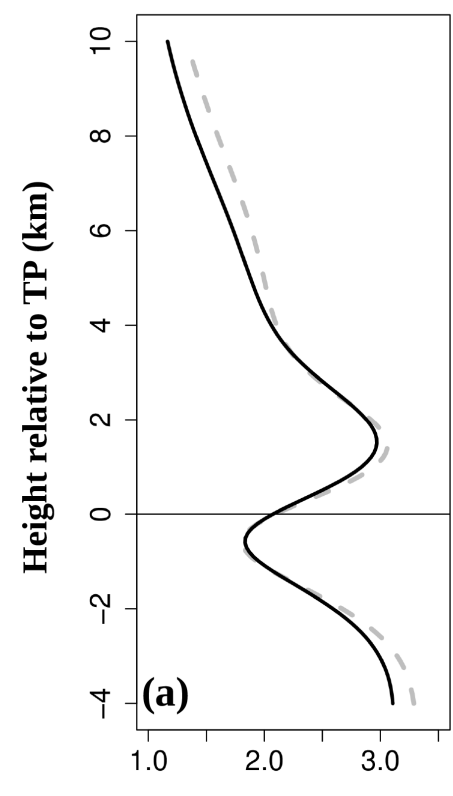

Temp. mean SD(K)

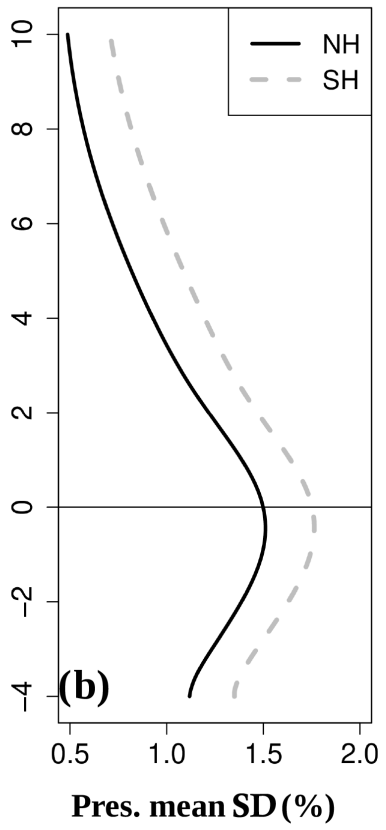

Figure 5. Vertical profiles of (a) temperature and (b) pressure timemean RWP activity within $40-60^{\circ}$ latitude, calculated as the mean standard deviation of w4-8 daily anomalies and relative to the zonal-mean tropopause height.

Recent studies using meridional winds and the Hilbert transform have shown the benefits of refining the methodology with semi-geostrophic coordinate adjustment (Wolf and Wirth, 2015, 2017) and even using wave activity flux (Takaya and Nakamura, 2001) for RWP diagnostics and optimizing the envelope's shape. Our approach to describe the phase and envelope propagation of an RWP in Fig. 6, with the only use of relative pressure anomalies from gridded GNSS-RO and the Hilbert transform over a limited wavenumber range (5-8), is quite simplistic in comparison but enough to get a good qualitative view. We concentrate now on the first RWP in Fig. 6 (27 February to 7 March 2009), exploring how its pressure and temperature structures evolve in the UTLS. Longitude-height snapshots of this RWP are presented at $2 \mathrm{~d}$ intervals in Fig. 7.

The RWP pressure anomalies in Fig. 7 (top row) are centered around the tropopause level, while the temperature anomalies (bottom row) have a clear separation between the UT and LS and a noisier appearance. This is in agreement with the results from the zonal-mean perspective from Figs. 1 and 2. The RWP pressure anomalies have a near-global coverage near the tropopause at almost all times, despite having segments of very low amplitudes: e.g., $\sim 60^{\circ} \mathrm{E}$ in 1 March 2009 and $\sim 120^{\circ} \mathrm{E}$ in 3 March 2009 in Fig. 7, top row. The zonal extent of the RWP pressure anomalies decreases away from the tropopause: e.g., compare pressure anomalies at 10 and $16 \mathrm{~km}$ in Fig. 7. The choice of a specific vertical level (a) Wave packet

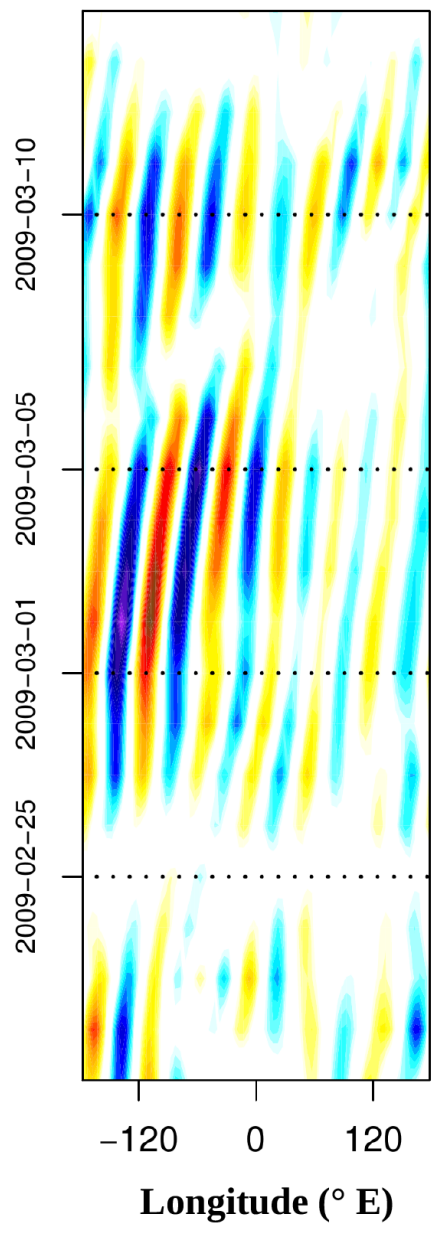

(b) Envelope

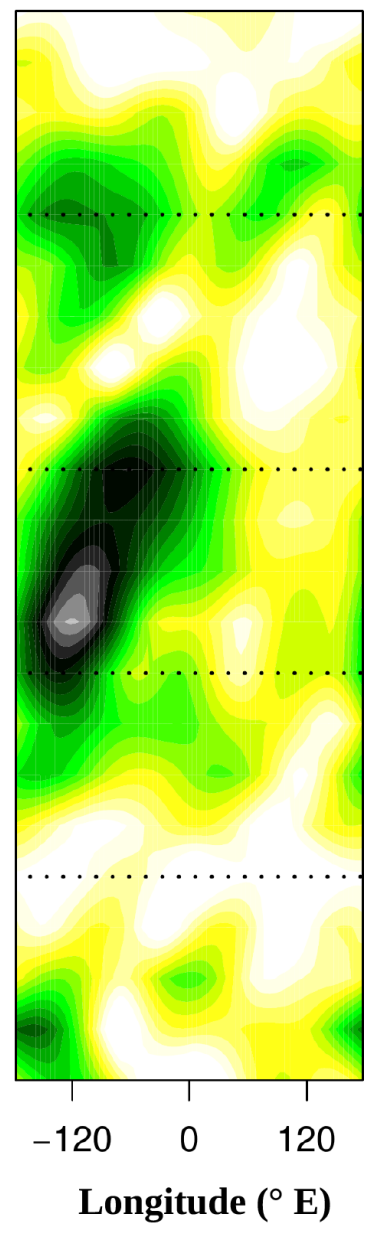

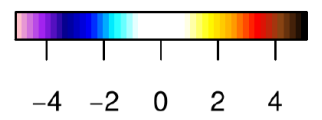

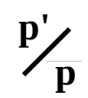

(\%)

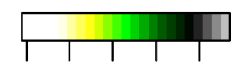

Figure 6. (a) Hovmöller diagram of RWP pressure anomalies (w58 ) at zonal-mean tropopause level from the end of February to the beginning of March 2009, averaged for $40-60^{\circ}$ N. (b) Corresponding envelope of the RWP anomalies.

to extract the RWP's envelope could affect the extent of the diagnosed RWP (see Discussion, Sect. 5).

Looking at the individual phases of the RWP pressure anomalies, the same troughs and ridges can be recognized going from $6 \mathrm{~km}$ up to $\sim 20 \mathrm{~km}$ height in Fig. 7 (top row). A slight westward tilt with height can be spotted in the stratosphere, which is typical of Rossby waves, although the RWP pressure structures in the UTLS can be considered almost barotropic. From the RWP pressure anomalies in Fig. 7 (top row) it can be concluded that RWPs form a direct dynamical connection between the UT and the stratosphere.

The RWP temperature anomalies in Fig. 7 (bottom row), apart from the break around tropopause level, show an outof-phase behavior between the UT and LS: troughs (blue 

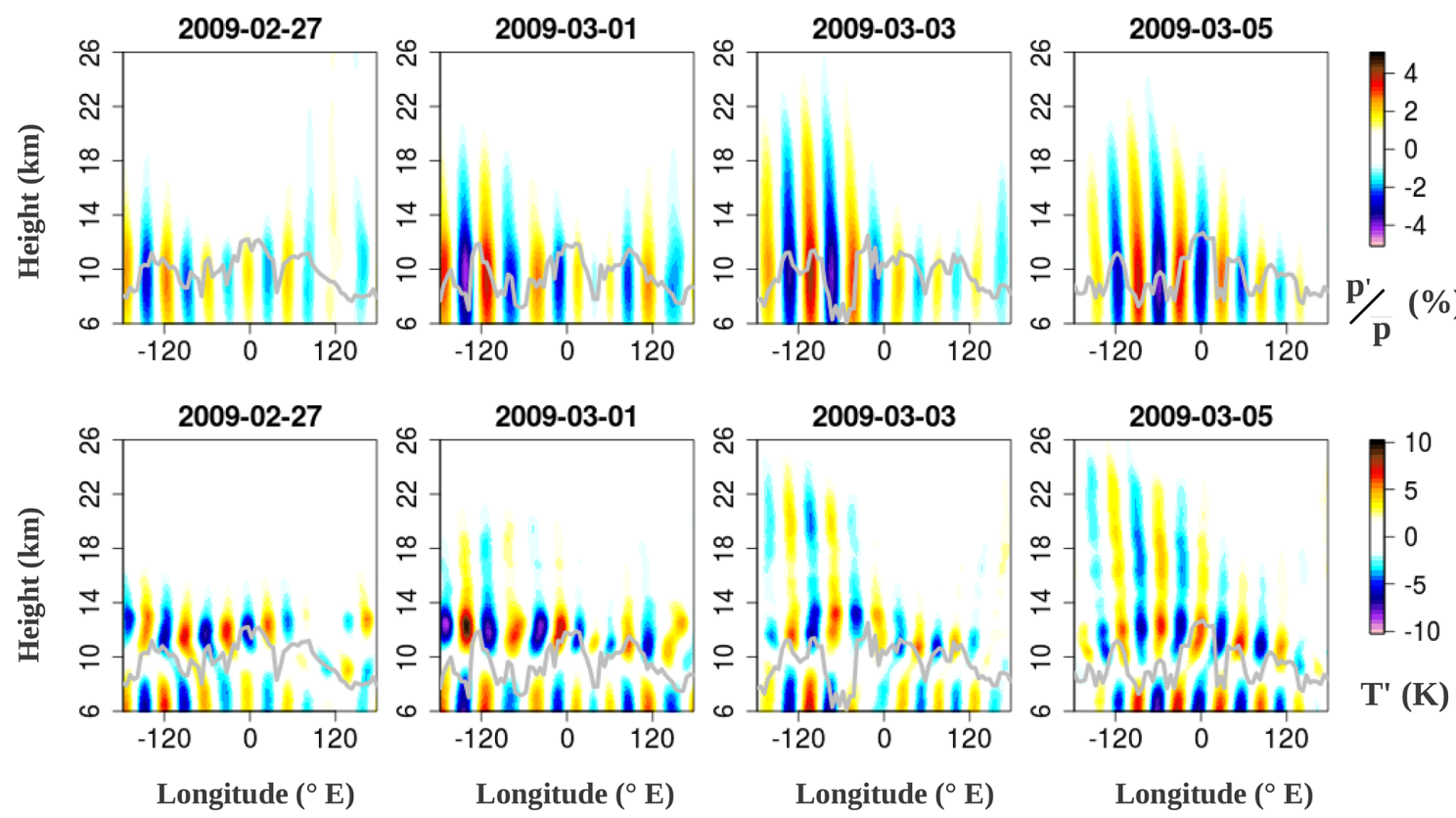

Figure 7. Longitude-height snapshots of (top row) pressure and (bottom row) temperature anomalies of the RWP from Fig. 6 at $50^{\circ}$ N. The grey line denotes the gridded lapse-rate tropopause height.

phases in Fig. 7, top row) correspond to negative temperature anomalies in the troposphere and positive temperature anomalies in the stratosphere. This is expected from the meridional advection associated with the barotropic pressure anomalies and the inversion of the meridional temperature gradient between the UT and LS (Volkmar Wirth, personal communication, 2019). However, the meridional temperature gradients in the lowermost stratosphere are of low magnitude, so it is not clear why the RWP temperature anomalies maximize so close to the tropopause and not higher up. Pilch Kedzierski et al. (2017) also reported wave amplitudes maximizing within a short height range close to the tropopause.

The longitude-height structures of RWP pressure and temperature anomalies in the UTLS from Fig. 7 show a high resemblance with those reported by Hakim (2005) (e.g., see their Fig. 7), although they used the leading empirical orthogonal functions (EOFs) of meridional winds and temperature. These structures are strongly related to forecast error propagation (Hakim, 2005), with wind errors maximizing near the tropopause, while temperature errors maximize near the surface. It is possible to collocate forecast error directly with observed RWP structures like those we show in Fig. 7, another interesting topic for a future study.

Recent case studies about extreme Arctic cyclones (Tao et al., 2017a, b) highlighted the influence of strong positive $\mathrm{PV}$ anomalies in the LS with a warm core in the deepening of the surface cyclones, which would be consistent with the presence of an RWP with UTLS pressure and temperature structures such as those depicted in Fig. 7, a high-amplitude trough in this case.

Odell et al. (2013) noted large stratospheric geopotential height tendencies during the 1993 Braer storm, the deepest extratropical low on record with $914 \mathrm{hPa}$ minimum surface pressure. A study of 60 severe European windstorms (Pirret et al., 2017) found that in 20 cases the stratosphere contributed $10 \%$ or more to their deepening. The pressure tendency equation used in Pirret et al. (2017) used the $100 \mathrm{hPa}$ level $(\sim 16 \mathrm{~km})$ as the upper boundary, with the stratospheric contribution being dependent on geopotential height tendencies at this boundary (their $\mathrm{d} P h i$ term). The presence of a high-amplitude RWP in the UTLS can significantly affect geopotential anomalies at the $100 \mathrm{hPa}$ level, as our results show (Fig. 7, top row; also Fig. 2 indicates pressure anomalies reaching this level very often). Therefore we hypothesize that $100 \mathrm{hPa}$ geopotential height tendencies (used to calculate the stratospheric contribution to surface cyclone deepening) could be very sensitive to the presence of RWP structures in the UTLS.

\subsection{The 2010 Russian heat wave case}

Recent studies have shown that in NH summer and with a specific setting of the waveguide(s), resonant conditions appear and drive high-amplitude waves which become quasistationary, with wavenumbers in the w6-8 range (Petoukhov et al., 2013; Kornhuber et al., 2017, 2019). This process has been linked to the 2003, 2010, 2015 and 2018 heat waves in Europe and Russia. Is the appearance of RWPs in the UTLS any different under these special conditions? Throughout this 
(a) Wave packet
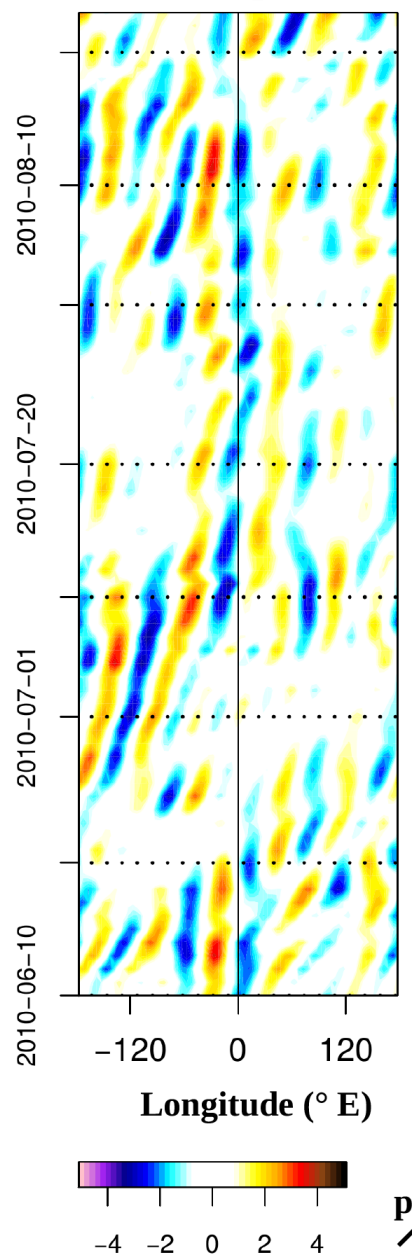

(b) Envelope

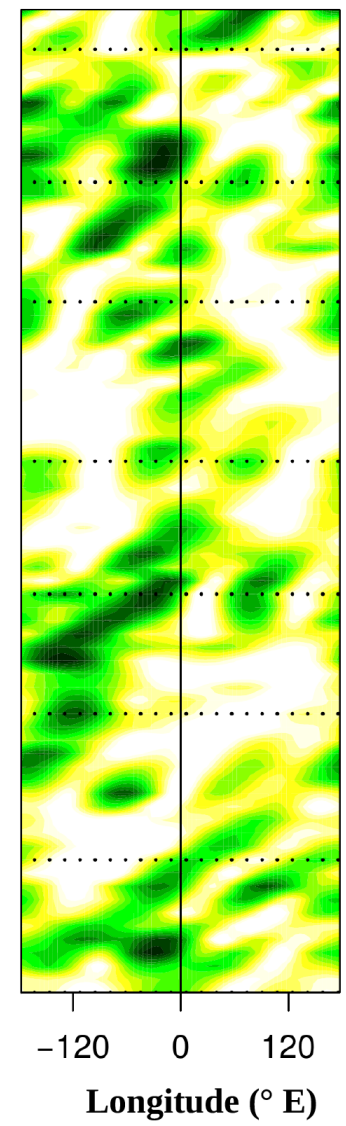

(\%)

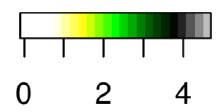

Figure 8. (a) Hovmöller diagram of RWP pressure anomalies (w48 ) at zonal-mean tropopause level for June-July-August 2010, averaged for $50-60^{\circ} \mathrm{N}$. (b) Corresponding envelope of the RWP anomalies in (a). Horizontal dotted lines mark the 1st, 10th and 20th days of the month.

section we will seek an answer by repeating the diagnostics of Sect. 4.1 for the summer 2010 Russian heat wave.

Figure 8a shows the evolution of RWP pressure anomalies (w4-8) during summer 2010 and Fig. 8b their corresponding envelope calculated with the Hilbert transform. It can be observed in Fig. 8b that the RWP envelope goes around the globe one and a half times between 10 June and 10 July. Eastward phase propagation is generally visible throughout this period, except for the time when the RWP is around $0^{\circ}$ longitude, where the phases become nearly stationary (Fig. 8a). Between 10 July and 10 August, a trough (blue) sets in around $0^{\circ}$ and barely moves, with ridges constantly present to its west and east. The ridge around $\sim 30-45^{\circ} \mathrm{E}$ is present nearby Moscow during this time. It is not until 20 August when a trough passes over Moscow's longitude.

Rather than one RWP expanding and becoming stationary, the envelope evolution in Fig. 8b suggests recurrent Atlantic RWPs with eastward group propagation terminating over 0 $60^{\circ} \mathrm{E}$, with near-zero phase speeds over this region between 10 July and 10 August. The amplitudes of the multiple RWPs in Fig. 8 are not higher than those of the typical winter case in Fig. 6, meaning that RWP anomalies in the UTLS need not be very strong to cause extreme events on the surface. The summer 2010 case is unusual for the low phase speeds around $0^{\circ}$ longitude and the recurrence of RWPs coming into the $0-60^{\circ} \mathrm{E}$ region, giving the event a much longer duration.

In Fig. 9 we present longitude-height snapshots of the summer 2010 RWPs. Given the longer event duration, we take a total of eight snapshots at $4 \mathrm{~d}$ intervals. RWP pressure anomalies in the UTLS (1st and 3rd rows) are maximized near the tropopause level and are quasi-barotropic, while the RWP temperature anomalies (second and fourth rows) maximize above the tropopause with a clear break between the UT and LS signals. No qualitative differences are found in the UTLS structures of RWPs between the typical winter case (Fig. 7) and the recurrent RWPs with stationary phases during the summer 2010 Russian heat wave (Fig. 9). A similar analysis of longitude-height snapshots during the 2015 European heat wave leads to the same qualitative conclusions (not shown) in terms of RWP pressure and temperature structures in the UTLS.

We conclude that the general behavior of RWPs across the UTLS, dynamically and thermally, is the same in normal winter conditions (Figs. 6 and 7 in Sect. 4.1) and summer under quasi-resonant waveguide setting (Figs. 8 and 9 in Sect. 4.2). The only differing factors during the summer 2010 heat wave are the low RWP phase speeds and their recurrence, although their UTLS appearance is alike to typical winter RWPs.

\subsection{Climatological statistics of RWP longitude-height structures}

\subsubsection{Vertical scale analysis}

Figures 7 and 9 showed the difference between the RWP appearance in terms of pressure and temperature anomalies: whereas pressure anomalies have a long vertical wavelength, temperature anomalies show a break around the tropopause and opposite phases in the UT and LS. Therefore it is expected that the vertical scale of RWP temperature anomalies is significantly shorter than that of RWP pressure anomalies, especially in the UTLS. To quantify this, we take all longitude-height snapshots of RWP (w4-8) anomalies between 40 and $60^{\circ}$ latitude, computing Fourier power spectra in the vertical direction between 6 and $36 \mathrm{~km}$ height. The resulting average Fourier power spectra for both hemispheres are shown in Fig. 10a.

Temperature (solid blue and dashed cyan lines) and pressure (red and dashed orange lines) vertical power spectra 

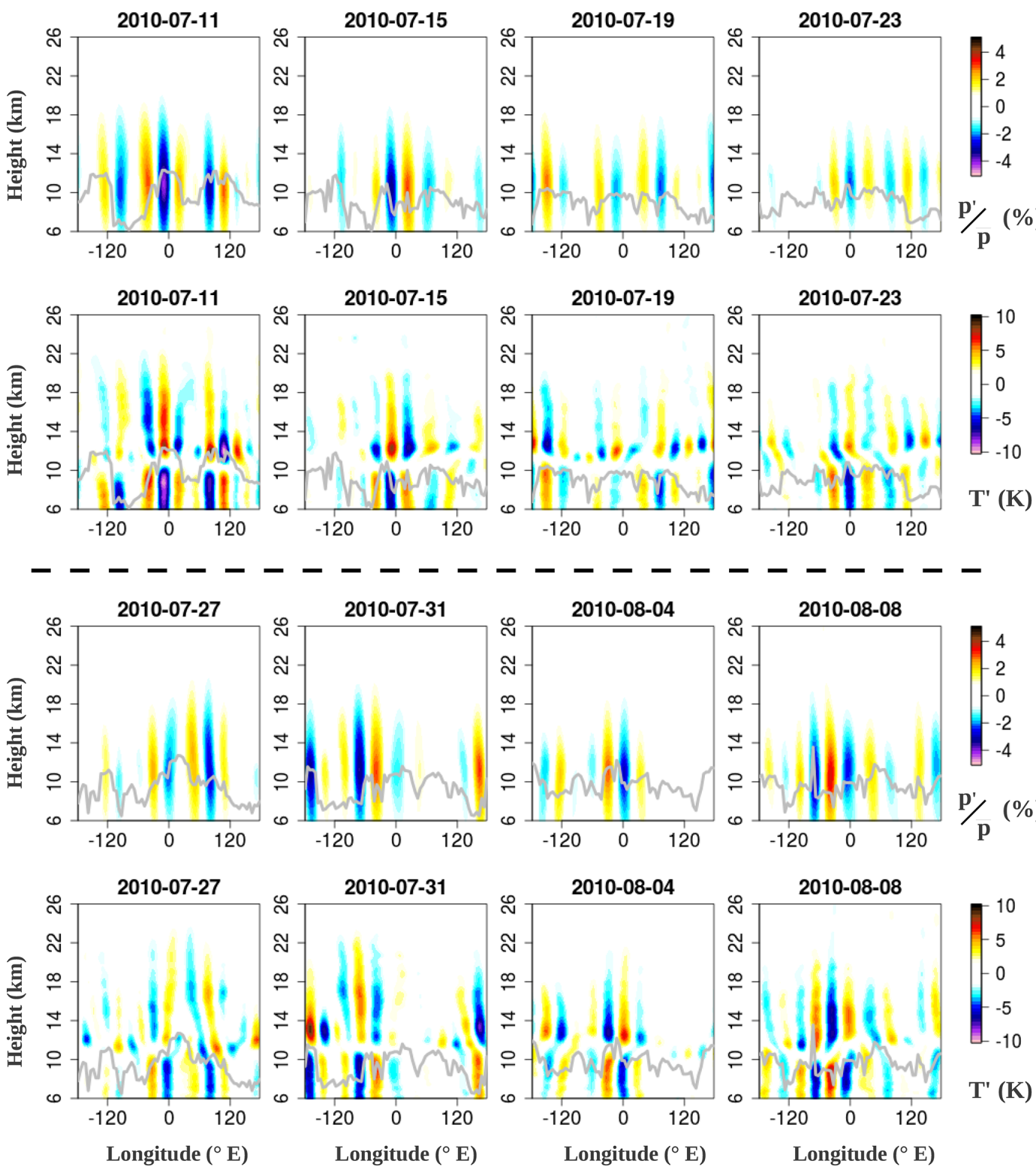

Figure 9. Longitude-height snapshots of (fist and third rows) pressure and (second and fourth rows) temperature anomalies of the RWP from Fig. 8 at $55^{\circ} \mathrm{N}$. The grey line denotes the gridded lapse-rate tropopause height.

for $\mathrm{NH}$ and SH show very little interhemispheric differences in Fig. 10a. Both RWP temperature and pressure anomalies (w4-8) have increasing power towards longer vertical wavelengths. The pressure power spectra show the steepest increase in power for vertical wavelengths $>10 \mathrm{~km}$, with a still noticeable amount of power between 5 and $10 \mathrm{~km}$ vertical wavelengths. Temperature vertical power spectra show the steepest increase in power at wavelengths $>5 \mathrm{~km}$ and a noticeable amount of power between 3 and $5 \mathrm{~km}$ wavelengths. Also, the integrated power of $1.5-3 \mathrm{~km}$ vertical wavelengths might be of significance in the temperature spectrum. Unlike pressure, the temperature structures of RWPs have a relatively large amount of power at vertical wavelengths $3-10 \mathrm{~km}$ in their Fourier spectra, and those are the vertical wavelengths where the power ratio between temperature and pressure is highest in Fig. 10b.

We repeated Fig. 10 selecting the same zonal wavenumbers (4-8) and additionally filtering in the time dimension to obtain 2-20 d eastward-propagating waves. This makes sure that neither stationary waves nor gravity waves (that oscillate at frequencies higher than the inertial frequency) are present. The result (see Fig. S4) is a near-identical spectrum.

In an atmosphere model, the vertical resolution (or the separation between model levels) needed to resolve wave temperature structures is $\sim 6$ times less than the wavelength. Most global climate models (GCMs) have vertical resolu- 
(a) Vertical power spectra for $40-60^{\circ}$ latitude

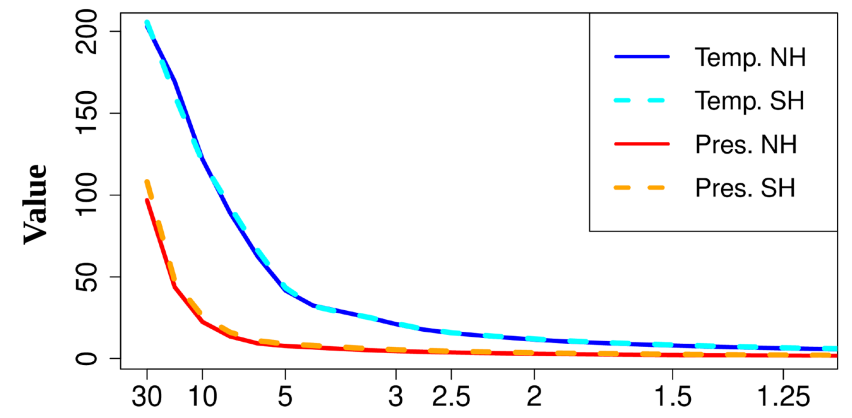

(b) Temperature-to-pressure power ratio

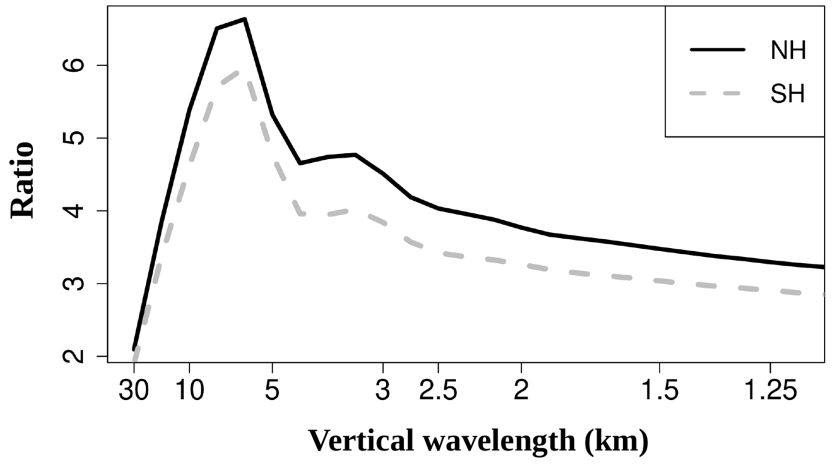

Figure 10. (a) Vertical power spectra of all RWP (w4-8) longitudeheight snapshots between $40-60^{\circ}$ latitude for both hemispheres. (b) Ratio of temperature and pressure power spectra.

tions coarser than $1 \mathrm{~km}$ in the UTLS, meaning that they will struggle to represent vertical wavelengths shorter than 6$8 \mathrm{~km}$. From the temperature vertical power spectra in Fig. 10a one could anticipate that RWP temperature structures in the UTLS such as Figs. 7 and 9 may be partially underrepresented by GCMs. In fact, GCMs, reanalyses and forecast models are known to produce temperature gradients in the tropopause region that are too smooth (Gettelman et al., 2010; Hegglin et al., 2010; Birner et al., 2006; Pilch Kedzierski et al., 2016b). The tendency of forecast models to smooth the tropopause with lead time (Gray et al., 2014) is partially compensated for by diabatic and parameterized processes in the modeled RWPs (Saffin et al., 2017).

A better understanding of RWP diabatic processes offers the chance of forecast improvement; therefore a variety of frameworks to analyze RWP evolution have been developed, mainly using reanalysis or forecast model output (e.g., Hoskins et al., 1985; Orlanski and Katzfey, 1991; NielsenGammon and Lefevre, 1996; Chang, 2001; Gray et al., 2014; Teubler and Riemer, 2016). The vertical structure of such processes is of high importance for RWP evolution, and analyses such as our Figs. 1, 5, 7, 9 or 10 would enable a comparison of model or reanalysis output directly with GNSS-RO observations. See Sect. 5 for a more detailed discussion.

\subsubsection{UT and LS out-of-phase temperature behavior}

Figures 7 and 9 indicate that the RWP thermal structures in the UT and LS have constantly opposing phases. If we take the zonal structures of RWP temperature anomalies $1.5 \mathrm{~km}$ above and $3 \mathrm{~km}$ below the zonal-mean tropopause as proxies for the LS and UT, respectively, in the case of Figs. 7 and 9 the correlation factors between the LS and UT would be close to $R=-1$. We test the prevalence of this behavior by creating probability density functions (PDFs) of the UTLS correlation factor $R$ against the wave's amplitude (as the standard deviation of the wave pressure anomalies at zonalmean tropopause level) for different wavenumber ranges and latitude bands in Fig. 11.

The PDF for midlatitude RWPs (w4-8, Fig. 11a) shows a general tendency for UT-LS anticorrelation of the temperature structures: higher densities (yellow-red colors) are increasingly packed towards $R=-1$ the higher the RWP pressure amplitude gets. The RWP temperature structures with opposite phases between the UT and LS are thus a typical behavior with very few exceptions as one can guess from the near-zero probability densities away from $R=-1$ in Fig. 11a.

Midlatitude planetary waves (w1-2, Fig. 11b) show a much more disperse distribution resulting in lower densities (grey-black colors) covering the whole $R$ range, evenly distributed especially at higher wave amplitudes. In Fig. 11b, the relative concentration towards $R=-1$ at lower wave amplitudes could be the result of the w1-2 components being part of an RWP, as explained next. One may consider an RWP with carrier w4 (e.g., the idealized example in Wolf and Wirth (2015); their Fig. 2d) and its zonal wavenumber Fourier spectrum: the RWP wavenumber spectrum peaks at w4, with decreasing power of the wavenumbers around it, and w1-2 are low-amplitude contributors to the RWP zonal structure the same way as w6-7. Higher-amplitude planetary waves, which cannot be part of RWPs, do not show such a tendency of the PDF to maximize near $R=-1$.

In the subtropics (Fig. 11c and d), both RWPs and planetary waves have lower amplitudes than their midlatitude counterparts. This is expected since Figs. 3 and 4 show very little transient (2-20 d period) wave activity there, and any wave amplitude of importance in Fig. 11c and d, which has not been filtered in the time dimension, should therefore come from stationary waves. The PDF for subtropical RWPs (w4-8, Fig. 11c) is concentrated at low amplitudes and shows very little shift towards $R=-1$, being spread throughout all $R$ values and looking nothing like the midlatitude PDF.

Planetary waves in the subtropics show a PDF packed near $R=-1$ (w1-2, Fig. 11d). As mentioned above, higher amplitudes of w1-2 belong to stationary signals, which translate into large-scale and long-lasting temperature anomalies of opposite sign in the subtropical UT and LS. A plausible explanation for Fig. 11d is represented by ENSO-related temperature anomalies in the UT and LS: they extend to the sub- 


\section{Anticorrelation of temp. signals between UT and LS}

(a) $40-60^{\circ} \mathrm{N}, \mathrm{w} 4-8$

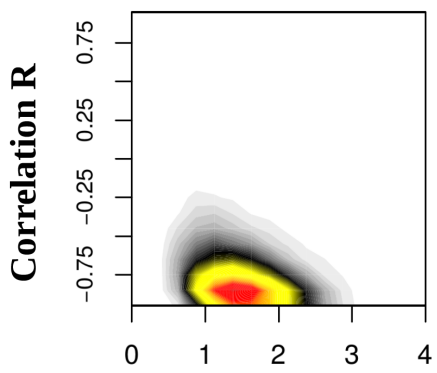

(c) $15-30^{\circ} \mathrm{N}, \mathrm{w} 4-8$

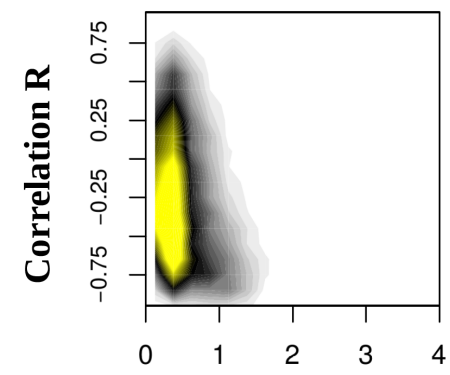

TP pressure SD(\%)

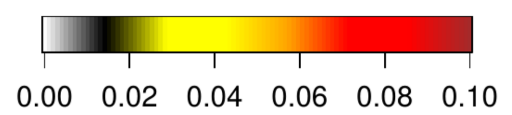

Prob. density

Figure 11. Probability density functions of the UT-LS temperature correlation vs. wave amplitudes for RWPs (w4-8, a, c) and planetary waves (w1-2, b, d) at midlatitudes $(\mathbf{a}, \mathbf{b})$ and the subtropics (c, d).

tropics, are long-lasting and their zonal scale fits well with a combination of zonal wavenumbers 1-2. Indeed Domeisen et al. (2019), their Fig. 5, showed opposite-signed UT and LS temperature regressions to the ENSO index. UT warm anomalies relate to increased convective latent-heat release and LS cold anomalies from the resulting enhanced largescale upwelling. These anomalies would combine into the anticorrelation shown in Fig. 11d.

We repeated the analysis of Fig. 11 on the $\mathrm{SH}$, finding nearly identical results (see Fig. S5 in the Supplement).

\subsubsection{Zonality of RWP envelopes: hemispheric comparison}

Early studies noticed the tendency of RWP envelopes to have a near-global scale in the $\mathrm{SH}$, while the $\mathrm{NH}$ shows a more zonally confined behavior (see Lau, 1979; Randel and Stanford, 1985, and references therein). Hemispheric comparisons by Chang (1999) and Souders et al. (2014b) with more

\section{Envelope power spectra for $40-60^{\circ}$ latitude}

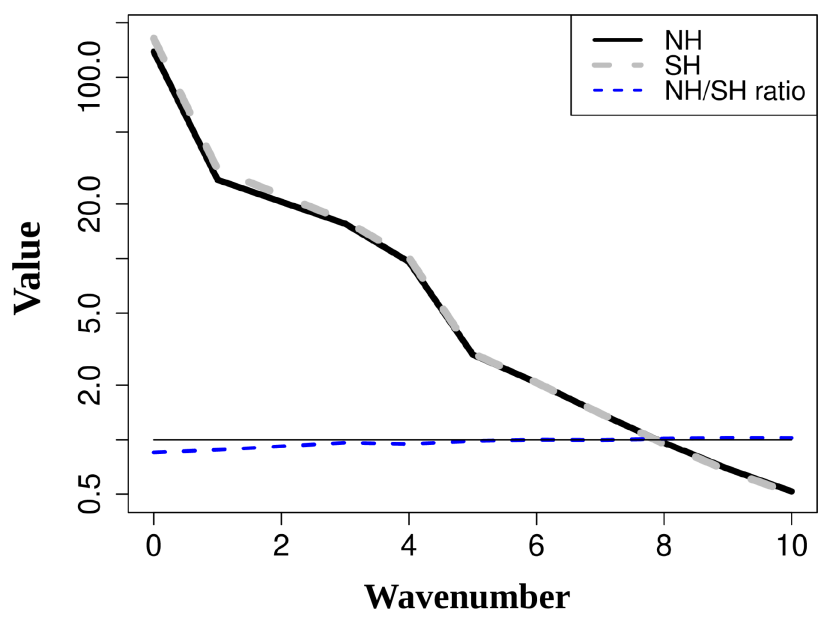

Figure 12. Average zonal wavenumber power spectra of RWP envelopes between 40 and $60^{\circ}$ latitude for $\mathrm{NH}$ (black) and $\mathrm{SH}$ (grey). The blue line denotes the $\mathrm{NH} / \mathrm{SH}$ ratio, with the thin black line showing a $1: 1$ ratio. As in Figs. 6 and 8, RWP envelopes are calculated at zonal-mean tropopause level.

advanced methods to define RWPs support these earlier findings. A Fourier analysis of the RWP envelope describes its zonal scale: the w0 component of the envelope corresponds to the global (or zonal-mean) extent, while w1 and higher components modulate how the envelope's amplitude varies regionally. Here we want to find out whether the $\mathrm{SH}$ has higher amounts of all envelopes' Fourier components or only of the longest ones. A comparison of the wavenumber power spectra of RWP envelopes from $\mathrm{NH}$ and $\mathrm{SH}$ midlatitudes is shown in Fig. 12.

A surprising similarity of the RWP envelope's wavenumber spectra for both hemispheres can be observed in Fig. 12 (solid black and dashed grey lines; also note the logarithmic $y$ scale). There is a steady exponential increase in power from w10 to w5, appearing as a line of constant slope in Fig. 12 between values from 0.5 to 2 . The increase in power from w4 to w1 is well above exponential, with a jump-like shift to values from 10 to $\sim 30$. The w0 component has the highest power $(\sim 150)$, which is expected because an RWP envelope is by definition always positive. It can be concluded that at tropopause level, w0-4 components markedly dominate the RWP envelope spectrum, with higher wavenumbers contributing marginally. This is observational support for previous studies which filtered out higher wavenumber components of the RWP envelope in their methodology: e.g., Wolf and Wirth (2015) eliminated the envelope's components $>$ w6 to avoid undesired small-scale wiggles in the envelope's shape.

To highlight where the differences between hemispheres are, we add the $\mathrm{NH} / \mathrm{SH}$ power ratio for each wavenumber as the dashed blue line in Fig. 12. It can be observed that 
for $w 3-10$, the $\mathrm{NH} / \mathrm{SH}$ ratio is very close to 1 : it ranges from 0.95 to 1.03 . The $\mathrm{NH} / \mathrm{SH}$ power ratio becomes lower at larger scales: 0.92 for w2, 0.88 for $w 1$ and 0.85 , the biggest hemispheric difference, for w0. This means that the longest wavenumber components ( $w 0$ and $w 1$ ) of RWP envelopes in the $\mathrm{NH}$ climatologically have $85 \%$ and $88 \%$ of the power found in the SH spectrum. Our results in Fig. 12, with the $\mathrm{SH}$ having more $\mathrm{w} 0$ and $\mathrm{w} 1$ in the RWP envelopes, are consistent with previous studies (Lau, 1979; Randel and Stanford, 1985; Chang, 1999; Souders et al., 2014b) in that the zonal extent of RWPs in the SH tends to be more global than in the $\mathrm{NH}$. In addition, we show that the amount of zonal variability of the envelope's amplitudes at a sub-planetary scale (w2 and higher) is basically the same in both hemispheres.

\section{Discussion}

The following points outline the importance of following the tropopause.

\subsection{For diagnosing RWP properties}

Throughout Sects. 3 and 4 our results depict RWP activity being centered around the tropopause height, following it at all times. At midlatitudes, the tropopause in the $\mathrm{NH}$ varies from $\sim 220-300 \mathrm{hPa}$ in winter to $\sim 175-250 \mathrm{hPa}$ in summer, while in the SH it varies from a fairly meridionally uniform $\sim 250 \mathrm{hPa}$ in winter to $\sim 200-280 \mathrm{hPa}$ in summer (Son et al., 2011). This has important implications for RWP diagnostics, which are usually performed with meridional winds at a fixed pressure level, which is not equidistant from the tropopause over latitude and season.

The most widely used pressure level for RWP diagnostics is $300 \mathrm{hPa}$, either for showcasing RWP envelope reconstruction or tracking methods (Zimin et al., 2003, 2006; Souders et al., 2014a; Wolf and Wirth, 2015, 2017), producing RWP climatologies or composites (Chang, 1999; Chang and Yu, 1999; Williams and Colucci, 2010; Souders et al., 2014b), or studying their predictability (Quinting and Vitart, 2019). The $250 \mathrm{hPa}$ level was also used by Glatt and Wirth (2014) and Grazzini and Vitart (2015) to study RWP properties and predictability in forecast models.

Generally a fixed threshold for RWP envelope amplitude is defined to determine whether an RWP is present and delimit the RWP's horizontal extent. Having the longitudeheight snapshots of RWP anomalies from Figs. 7 and 9 in mind (relative pressure RWP amplitudes being proportional to geostrophic meridional wind amplitudes, as explained in Sect. 2.4), the diagnosed RWP amplitude and extent would increase the closer the chosen vertical level is to the zonalmean tropopause. For a given pressure level, its distance from the tropopause level will vary seasonally and over latitude, introducing an aliasing factor if RWP properties are compared between summer and winter or NH and SH. Some- times this issue could even affect the number of diagnosed RWPs: if the pressure level is further from the tropopause than usual, some part of an RWP may show meridional winds below the threshold, which leads to fragmentation into two or more RWPs; or a low-amplitude RWP may not be detected at all, while at tropopause level the threshold is still exceeded. Meridional wind on the $2 \mathrm{PVU}$ surface (the so-called dynamical tropopause) is an available reanalysis product that is a perfect candidate to avoid the above-discussed aliasing effect on RWP properties.

Some methods make use of a threshold relative to the zonally averaged RWP envelope amplitude (e.g., Glatt and Wirth, 2014; Grazzini and Vitart, 2015; Quinting and Vitart, 2019). While solving the issue of pressure level distance to the tropopause, in this case an even bigger problem arises: the presence of stronger wave activity over a near-global longitude range would increase the threshold substantially, reducing the diagnosed RWP size (Wolf and Wirth, 2017). Also, in the case of a zonally localized RWP growing into a highamplitude and global wave due to resonant conditions (e.g., Petoukhov et al., 2013), a relative threshold would by contrast yield a shrinking RWP.

\subsection{For RWP energy budgets and fluxes}

RWP eddy kinetic energy (EKE) budgets are usually presented as vertical integrals, from the surface up to the upper boundary at $100 \mathrm{hpa}$ (Orlanski and Katzfey, 1991; Chang, 2000, 2001) or $50 \mathrm{hPa}$ (Orlanski and Sheldon, 1993). Additionally, Chang (2001) showed volume-averaged composites of RWP wave activity fluxes for the UTLS (400$200 \mathrm{hPa}$ ), noting that the baroclinic growth - barotropic decay paradigm of RWP life cycles has to be treated with caution when interpreting zonally localized waves. PV inversion diagnostics by, e.g., Nielsen-Gammon and Lefevre (1996) use 1000 and $100 \mathrm{hPa}$ as boundaries for the PV tendency equation, presenting different terms and compositing at a fixed pressure level (e.g., near $300 \mathrm{hPa}$ ).

Our results suggest that compositing energy budget terms and fluxes relative to the tropopause height, or even making separate budgets for the UT and LS, would add precision to the magnitude, location and role of each term. Winddependent terms would maximize near the tropopause, since our RWP pressure anomalies are proportional to geostrophic winds. The temperature-dependent terms would show high sensitivity to their location relative to the tropopause, since our RWP temperature signals maximize right above the tropopause and become very low right below it (see Figs. 1, 2, 5, 7 and 9). Averaging on pressure levels may lead to smoothing of the vertical structures of specific EKE budget or PV tendency terms. Tropopause-based averaging retains UTLS gradients as introduced by Birner et al. (2002) and Birner (2006) for radiosonde temperature structures.

Recent publications are already applying a tropopauserelative framework to study PV tendencies (e.g., Cavallo and 
Hakim, 2009; Saffin et al., 2017), compositing for cyclones and troughs and ridges. Such an approach combined with a refined method to localize RWP envelopes (see discussion above) could improve the understanding of the forcings involved in RWP life cycles and their interaction with the background flow, e.g., in 3-D composites similar to those presented by Chang (2001).

\subsection{For diagnosing wave trends}

Quantifying the waviness of the extratropical westerly flow is challenging (see review by Coumou et al., 2018): each measure may have a different physical meaning and different degrees of complexity for systematic implementation on reanalysis or model data. Waviness trends are inconsistent among different methodologies (e.g., Barnes, 2013; Coumou et al., 2015; Cattiaux et al., 2016). These studies have in common that they perform their analyses on fixed pressure levels (e.g., 500 and $250 \mathrm{hPa}$ for wave amplitudes - Barnes, 2013 and Cattiaux et al., 2016 - or 850-250 hPa integrals for EKE trends - Coumou et al., 2015). Thus, they do not consider tropopause height biases in the models used for climate projections, which can be different for each model, potentially adding spread to the results. Neither do these studies account for modeled or reanalyzed tropopause height trends (e.g., tropospheric expansion leads to an increase in extratropical tropopause height over time with climate change). Interannual variability of the extratropical tropopause height alone would introduce aliasing effects over the diagnosed waviness on a fixed pressure level. Wave amplitudes diagnosed on the 2 PVU surface, as our results show, would always be equidistant from the location of the maximum in wave activity in the jet stream, thus providing the fairest trend measure and model intercomparison in terms of wave amplitudes.

The issue of tropopause trends was already noted for the attribution of stratospheric residual circulation trends by Oberländer-Hayn et al. (2016). We add that, since RWPs show a quasi-barotropic structure throughout the UTLS (See Figs. 7 and 9), RWP phase speeds in principle are not subject to the aliasing effect of varying tropopause distance from the diagnosed pressure level, so phase speed trends like those studied by Coumou et al. (2015) are not affected by this issue.

\section{Conclusions}

Our study is a first attempt to describe RWP properties globally with the sole use of GNSS-RO observations, focusing on both hemispheres' extratropical UTLS. Observational knowledge about RWPs such as the one presented here is much needed for comparison and interpretation of climate model and reanalysis output, especially with the current scientific effort to better understand storm track and jet stream responses to climate change. Our results are relevant for stratosphere-troposphere coupling: they indicate systematic RWP activity propagation into the stratosphere, which markedly increases during SSWs, in addition to the wellknown RWP driving of tropospheric weather. We summarize our main findings below.

1. RWP activity follows the zonal-mean tropopause level and maximizes around it during all seasons (Figs. 1, 2, 5, 7 and 9). RWP pressure anomalies tend to be centered at the tropopause, with decreasing amplitude away from it. RWP temperature anomalies maximize right above the tropopause, with a contrasting minimum right below the tropopause.

2. We note frequent RWP propagation into the stratosphere, sometimes beyond $20 \mathrm{~km}$ height in the $\mathrm{NH}$, which mostly manifests itself for wavenumbers 4 and 5 (Figs. 1 and 2). Enhanced vertical propagation of RWP activity coincided with the SSWs of 2009, 2010 and 2013 (see Figs. S1, S2 and S3). We will explore this further in an upcoming study, given the importance of the lower stratosphere in modulating the onset and downward propagation of SSWs.

3. Since RWP activity constantly follows the tropopause, we note that the use of fixed pressure levels for RWP diagnostics or wave trends (e.g., typically $300 \mathrm{hPa}$ ) may induce some aliasing in the resulting quantities, since it is not equidistant from the tropopause over time and latitude. RWPs would lose envelope extent and/or amplitude the further the pressure level is from the tropopause. We suggest using the $2 \mathrm{PVU}$ surface to avoid this (see discussions in Sect. 5).

4. In addition to gridded temperature, our study is the first to analyze GNSS-RO (relative) pressure anomalies, which are conveniently proportional to geostrophic meridional wind wave amplitudes (see Sect. 2.4). The filtered RWP pressure signals show much better continuity (i.e., no breaks in their vertical structure) and less noisiness in the UTLS and the stratosphere, compared to temperature wave signals (Figs. 1, 2, 7 and 9). This new approach shows many benefits for studying extratropical wave propagation with GNSS-RO data, especially in the vertical direction.

5. The dynamical and thermal appearance of RWPs in the UTLS is generally similar in different seasons and waveguide settings: no qualitative differences were observed after comparing a typical winter RWP with RWP activity during the 2010 Russian heat wave where resonant conditions were present (see Sects. 4.1 and 4.2).

6. Overall, RWPs in the SH show a preference for lower carrier wavenumbers compared to the NH (Figs. 3 and 4). In terms of envelope properties, the $\mathrm{SH}$ shows a higher amount of the longest scales compared to the $\mathrm{NH}$ 
(w0, w1; Fig. 12). This is in good agreement with previous observational and model studies. Apart from the above, we found that RWP properties in the UTLS are generally very similar across hemispheres.

Code and data availability. The datasets used for this publication were downloaded from the following web pages upon registration: ERA-Interim data - http://apps.ecmwf.int/datasets/ data/interim-full-daily/levtype=pl/ (last access: 15 July 2020, ECMWF, 2020; Dee et al., 2011, https://doi.org/10.1002/qj.828); GNSS-RO data from different satellite missions http://cdaac-www.cosmic.ucar.edu/cdaac/products.html (last access: 15 July 2020, CDAAC, 2020). See Sect. 2.1 for full data description and references. The " $R$ " mathematical software is available here: https://www.r-project.org/ (last access: 15 July 2020, R Core Team, 2015). The "NCL" space-time filter to extract waves is available here: https://www.ncl.ucar.edu/Document/ Functions/User_contributed/kf_filter.shtml (last access: 15 July 2020, Schreck, 2009).

Supplement. The supplement related to this article is available online at: https://doi.org/10.5194/acp-20-11569-2020-supplement.

Author contributions. RPK designed the methodology, performed the analyses, produced all figures and wrote the paper. $\mathrm{KM}$ and $\mathrm{KB}$ contributed with ideas and commented on the paper.

Competing interests. The authors declare that they have no conflict of interest.

Acknowledgements. This study was funded by the GEOMAR Helmholtz Centre for Ocean Research in Kiel. We thank the ECMWF data server for the freely available ERA-Interim data. We also thank UCAR for the reprocessing and availability of GNSSRO data from all satellite missions. Discussions with and comment from Tim Kruschke, Volkmar Wirth and Daniela Domeisen at the early stages of the study are deeply appreciated.

Financial support. The article processing charges for this openaccess publication were covered by a Research Centre of the Helmholtz Association.

Review statement. This paper was edited by Geraint Vaughan and reviewed by two anonymous referees.

\section{References}

Alexander, P., de la Torre, A., Llamedo, P., and Hierro, R.: Precision estimation in temperature and refractivity profiles retrieved by GPS radio occultations, J. Geophys. Res.-Atmos., 119, 86248638, https://doi.org/10.1002/2013JD021016, 2014.

Alexander, S. P. and Shepherd, M. G.: Planetary wave activity in the polar lower stratosphere, Atmos. Chem. Phys., 10, 707-718, https://doi.org/10.5194/acp-10-707-2010, 2010.

Alexander, S. P., Tsuda, T., Kawatani, Y., and Takahashi, M.: Global distribution of atmospheric waves in the equatorial upper troposphere and lower stratosphere: COSMIC observations of wave mean flow interactions, J. Geophys. Res.-Atmos., 113, D24115, https://doi.org/10.1029/2008JD010039, 2008.

Andrews, D. G., Holton, J. R., and Leovy, C. B.: Middle atmosphere dynamics, International Geophysics Series, Vol. 40, Academic Press, New York, 1987.

Anthes, R. A.: Exploring Earth's atmosphere with radio occultation: contributions to weather, climate and space weather, Atmos. Meas. Tech., 4, 1077-1103, https://doi.org/10.5194/amt-4-10772011, 2011.

Anthes, R. A., Bernhardt, P. A., Chen, Y., Cucurull, L., Dymond, K. F., Ector, D., Healy, S. B., Ho, S.-P., Hunt, D. C., Kuo, Y.H., Liu, H., Manning, K., McCormick, C., Meehan, T. K., Randel, W. J., Rocken, C., Schreiner, W. S., Sokolovskiy, S. V., Syndergaard, S., Thompson, D. C., Trenberth, K. E., Wee, T.K., Yen, N. L., and Zeng, Z.: The COSMIC/FORMOSAT-3 Mission: Early Results, B. Am. Meteorol. Soc., 89, 313-334, https://doi.org/10.1175/BAMS-89-3-313, 2008.

Barnes, E. A.: Revisiting the evidence linking Arctic amplification to extreme weather in midlatitudes, Geophys. Res. Lett., 40, 4734-4739, https://doi.org/10.1002/grl.50880, 2013.

Baumgart, M., Riemer, M., Wirth, V., Teubler, F., and Lang, S. T. K.: Potential Vorticity Dynamics of Forecast Errors: A Quantitative Case Study, Mon. Weather Rev., 146, 1405-1425, https://doi.org/10.1175/MWR-D-17-0196.1, 2018.

Beyerle, G., Schmidt, T., Michalak, G., Heise, S., Wickert, J., and Reigber, C.: GPS radio occultation with GRACE: Atmospheric profiling utilizing the zero difference technique, Geophys. Res. Lett., 32, L13806, https://doi.org/10.1029/2005GL023109, 2005.

Beyerle, G., Grunwaldt, L., Heise, S., Köhler, W., König, R., Michalak, G., Rothacher, M., Schmidt, T., Wickert, J., Tapley, B. D., and Giesinger, B.: First results from the GPS atmosphere sounding experiment TOR aboard the TerraSAR-X satellite, Atmos. Chem. Phys., 11, 6687-6699, https://doi.org/10.5194/acp11-6687-2011, 2011.

Birner, T.: Fine-scale structure of the extratropical tropopause region, J. Geophys. Res.-Atmos., 111, D04104, https://doi.org/10.1029/2005JD006301, 2006.

Birner, T., Dörnbrack, A., and Schumann, U.: How sharp is the tropopause at midlatitudes?, Geophys. Res. Lett., 29, 1700, https://doi.org/10.1029/2002GL015142, 2002.

Birner, T., Sankey, D., and Shepherd, T. G.: The tropopause inversion layer in models and analyses, Geophys. Res. Lett., 33, L14804, https://doi.org/10.1029/2006GL026549, 2006.

Blackmon, M. L. and White, G. H.: zonal Wavenumber Characteristics of Northern Hemisphere Transient Eddies, J. Atmos. Sci., 39, 1985-1998, https://doi.org/10.1175/15200469(1982)039<1985:ZWCONH>2.0.CO;2, 1982. 
Cattiaux, J., Peings, Y., Saint-Martin, D., Trou-Kechout, N., and Vavrus, S. J.: Sinuosity of midlatitude atmospheric flow in a warming world, Geophys. Res. Lett., 43, 8259-8268, https://doi.org/10.1002/2016GL070309, 2016.

Cavallo, S. M. and Hakim, G. J.: Potential Vorticity Diagnosis of a Tropopause Polar Cyclone, Mon. Weather Rev., 137, 1358-1371, https://doi.org/10.1175/2008MWR2670.1, 2009.

CDAAC (COSMIC Data Analysis and Archive Center): CDAAC Data Products, available at: http://cdaac-www.cosmic.ucar.edu/ cdaac/products.html, last access: 15 July 2020.

Chang, E. K. M.: Characteristics of Wave Packets in the Upper Troposphere. Part II: Seasonal and Hemispheric Variations, J. Atmos. Sci., 56, 1729-1747, https://doi.org/10.1175/15200469(1999)056<1729:COWPIT>2.0.CO;2, 1999.

Chang, E. K. M.: Wave Packets and Life Cycles of Troughs in the Upper Troposphere: Examples from the Southern Hemisphere Summer Season of 1984/85, Mon. Weather Rev., 128, 25-50, https://doi.org/10.1175/15200493(2000)128<0025:WPALCO>2.0.CO;2, 2000.

Chang, E. K. M.: The Structure of Baroclinic Wave Packets, J. Atmos. Sci., 58, 1694-1713, https://doi.org/10.1175/15200469(2001)058<1694:TSOBWP>2.0.CO;2, 2001.

Chang, E. K. M. and Yu, D. B.: Characteristics of Wave Packets in the Upper Troposphere. Part I: Northern Hemisphere Winter, J. Atmos. Sci., 56, 1708-1728, https://doi.org/10.1175/15200469(1999)056<1708:COWPIT>2.0.CO;2, 1999.

Chang, E. K. M., Lee, S., and Swanson, K. L.: Storm Track Dynamics, J. Clim., 15, 2163-2183, https://doi.org/10.1175/15200442(2002)015<02163:STD>2.0.CO;2, 2002.

Chang, E. K. M., Guo, Y., and Xia, X.: CMIP5 multimodel ensemble projection of storm track change under global warming, J. Geophys. Res.-Atmos., 117, D23118, https://doi.org/10.1029/2012JD018578, 2012.

Charney, J. G. and Drazin, P. G.: Propagation of planetary-scale disturbances from the lower into the upper atmosphere, J. Geophys. Res., 66, 83-109, https://doi.org/10.1029/JZ066i001p00083, 1961.

Coumou, D., Lehmann, J., and Beckmann, J.: The weakening summer circulation in the Northern Hemisphere mid-latitudes, Science, 348, 324-327, https://doi.org/10.1126/science.1261768, 2015.

Coumou, D., Di Capua, G., Vavrus, S., Wang, L., and Wang, S.: The influence of Arctic amplification on mid-latitude summer circulation, Nat. Commun., 9, 2959, https://doi.org/10.1038/s41467018-05256-8, 2018.

Danzer, J., Foelsche, U., Scherllin-Pirscher, B., and Schwärz, M.: Influence of changes in humidity on dry temperature in GPS RO climatologies, Atmos. Meas. Tech., 7, 2883-2896, https://doi.org/10.5194/amt-7-2883-2014, 2014.

Dee, D. P., Uppala, S. M., Simmons, A. J., Berrisford, P., Poli, P., Kobayashi, S., Andrae, U., Balmaseda, M. A., Balsamo, G., Bauer, P., Bechtold, P., Beljaars, A. C. M., van de Berg, L., Bidlot, J., Bormann, N., Delsol, C., Dragani, R., Fuentes, M., Geer, A. J., Haimberger, L., Healy, S. B., Hersbach, H., Hólm, E. V., Isaksen, L., Kållberg, P., Köhler, M., Matricardi, M., McNally, A. P., Monge-Sanz, B. M., Morcrette, J.-J., Park, B.-K., Peubey, C., de Rosnay, P., Tavolato, C., Thépaut, J.-N., and Vitart, F.: The ERA-Interim reanalysis: configuration and performance of the data assimilation system, Q. J. Roy. Meteorol. Soc., 137, 553597, https://doi.org/10.1002/qj.828, 2011.

Dirren, S., Didone, M., and Davies, H. C.: Diagnosis of "forecastanalysis" differences of a weather prediction system, Geophys. Res. Lett., 30, 2060, https://doi.org/10.1029/2003GL017986, 2003.

Domeisen, D. I., Garfinkel, C. I., and Butler, A. H.: The Teleconnection of El Niño Southern Oscillation to the Stratosphere, Rev. Geophys., 57, 5-47, https://doi.org/10.1029/2018RG000596, 2019.

Domeisen, D. I. V., Martius, O., and Jiménez-Esteve, B.: Rossby Wave Propagation into the Northern Hemisphere Stratosphere: The Role of Zonal Phase Speed, Geophys. Res. Lett., 45, 2064 2071, https://doi.org/10.1002/2017GL076886, 2018.

ECMWF: ERA Interim, Daily, available at: http://apps.ecmwf.int/ datasets/data/interim-full-daily/levtype $=\mathrm{pl} /$, last access: 15 July 2020.

Flannaghan, T. J. and Fueglistaler, S.: The importance of the tropical tropopause layer for equatorial Kelvin wave propagation, J. Geophys. Res.-Atmos., 118, 5160-5175, https://doi.org/10.1002/jgrd.50418, 2013.

Gall, R.: A Comparison of Linear Baroclinic Instability Theory with the Eddy Statistics of a General Circulation Model, J. Atmos. Sci., 33, 349-373, https://doi.org/10.1175/15200469(1976)033<0349:ACOLBI>2.0.CO;2, 1976.

Gettelman, A., Hegglin, M. I., Son, S.-W., Kim, J., Fujiwara, M., Birner, T., Kremser, S., Rex, M., Añel, J. A., Akiyoshi, H., Austin, J., Bekki, S., Braesike, P., Brühl, C., Butchart, N., Chipperfield, M., Dameris, M., Dhomse, S., Garny, H., Hardiman, S. C., Jöckel, P., Kinnison, D. E., Lamarque, J. F., Mancini, E., Marchand, M., Michou, M., Morgenstern, O., Pawson, S., Pitari, G., Plummer, D., Pyle, J. A., Rozanov, E., Scinocca, J., Shepherd, T. G., Shibata, K., Smale, D., Teyssèdre, H., and Tian, W.: Multimodel assessment of the upper troposphere and lower stratosphere: Tropics and global trends, J. Geophys. Res.-Atmos., 115 , D00M08, https://doi.org/10.1029/2009JD013638, 2010.

Giannakaki, P. and Martius, O.: An Object-Based Forecast Verification Tool for Synoptic-Scale Rossby Waveguides, Weather Forecast., 31, 937-946, https://doi.org/10.1175/WAF-D-15-0147.1, 2016.

Glatt, I. and Wirth, V.: Identifying Rossby wave trains and quantifying their properties, Q. J. Roy. Meteorol. Soc., 140, 384-396, https://doi.org/10.1002/qj.2139, 2014.

Gorbunov, M. E., Benzon, H.-H., Jensen, A. S., Lohmann, M. S., and Nielsen, A. S.: Comparative analysis of radio occultation processing approaches based on Fourier integral operators, Radio Sci., 39, RS6004, https://doi.org/10.1029/2003RS002916, 2004.

Gray, S. L., Dunning, C. M., Methven, J., Masato, G., and Chagnon, J. M.: Systematic model forecast error in Rossby wave structure, Geophys. Res. Lett., 41, 2979-2987, https://doi.org/10.1002/2014GL059282, 2014.

Grazzini, F. and Vitart, F.: Atmospheric predictability and Rossby wave packets, Q. J. Roy. Meteorol. Soc., 141, 2793-2802, https://doi.org/10.1002/qj.2564, 2015.

Hajj, G. A., Ao, C. O., Iijima, B. A., Kuang, D., Kursinski, E. R., Mannucci, A. J., Meehan, T. K., Romans, L. J., de la Torre Juarez, M., and Yunck, T. P.: CHAMP and SAC-C atmospheric occultation results and intercomparisons, J. Geophys. Res.-Atmos., 109, D06109, https://doi.org/10.1029/2003JD003909, 2004. 
Hakim, G. J.: Vertical Structure of Midlatitude Analysis and Forecast Errors, Mon. Weather Rev., 133, 567, https://doi.org/10.1175/MWR-2882.1, 2005.

Hall, R., Erdélyi, R., Hanna, E., Jones, J. M., and Scaife, A. A.: Drivers of North Atlantic Polar Front jet stream variability, Int. J. Climatol., 35, 1697-1720, https://doi.org/10.1002/joc.4121, 2015.

Hayashi, V. and Golder, D. G.: Transient Planetary Waves Simulated by GFDL Spectral General Circulation Models. Part I: Effects of Mountains, J. Atmos. Sci., 40, 941-950, https://doi.org/10.1175/15200469(1983)040<0941:TPWSBG>2.0.CO;2, 1983.

Healy, S. B. and Eyre, J. R.: Retrieving temperature, water vapour and surface pressure information from refractiveindex profiles derived by radio occultation: A simulation study, Q. J. Roy. Meteorol. Soc., 126, 1661-1683, https://doi.org/10.1002/qj.49712656606, 2000.

Hegglin, M. I., Gettelman, A., Hoor, P., Krichevsky, R., Manney, G. L., Pan, L. L., Son, S.-W., Stiller, G., Tilmes, S., Walker, K. A., Eyring, V., Shepherd, T. G., Waugh, D., Akiyoshi, H., Añel, J. A., Austin, J., Baumgaertner, A., Bekki, S., Braesicke, P., Brühl, C., Butchart, N., Chipperfield, M., Dameris, M., Dhomse, S., Frith, S., Garny, H., Hardiman, S. C., Jöckel, P., Kinnison, D. E., Lamarque, J. F., Mancini, E., Michou, M., Morgenstern, O., Nakamura, T., Olivié, D., Pawson, S., Pitari, G., Plummer, D. A., Pyle, J. A., Rozanov, E., Scinocca, J. F., Shibata, K., Smale, D., Teyssèdre, H., Tian, W., and Yamashita, Y.: Multimodel assessment of the upper troposphere and lower stratosphere: Extratropics, J. Geophys. Res.-Atmos., 115, D00M09, https://doi.org/10.1029/2010JD013884, 2010.

Hirooka, T., Kuki, T., and Hirota, I.: An Intercomparison of Medium-Scale Waves in the Northern and Southern Hemispheres, J. Meteorol. Soc. Jpn. Ser. II, 66, 857-868, https://doi.org/10.2151/jmsj1965.66.6_857, 1988.

Ho, S.-P., Peng, L., and Vömel, H.: Characterization of the longterm radiosonde temperature biases in the upper troposphere and lower stratosphere using COSMIC and Metop-A/GRAS data from 2006 to 2014, Atmos. Chem. Phys., 17, 4493-4511, https://doi.org/10.5194/acp-17-4493-2017, 2017.

Hoskins, B. J. and Valdes, P. J.: On the Existence of Storm-Tracks, J. Atmos. Sci., 47, 1854-1864, https://doi.org/10.1175/15200469(1990)047<1854:OTEOST>2.0.CO;2, 1990.

Hoskins, B. J., James, I. N., and White, G. H.: The Shape, Propagation and Mean-Flow Interaction of Large-Scale Weather Systems, J. Atmos. Sci., 40, 1595-1612, https://doi.org/10.1175/15200469(1983)040<1595:TSPAMF>2.0.CO;2, 1983.

Hoskins, B. J., McIntyre, M. E., and Robertson, A. W.: On the use and significance of isentropic potential vorticity maps, Q. J. Roy. Meteorol. Soc., 111, 877-946, https://doi.org/10.1002/qj.49711147002, 1985.

Hovmöller, E.: The Trough-and-Ridge diagram, Tellus A, 1, 62-66, https://doi.org/10.1111/j.2153-3490.1949.tb01260.x, 1949.

Karpechko, A. Y., Hitchcock, P., Peters, D. H. W., and Schneidereit, A.: Predictability of downward propagation of major sudden stratospheric warmings, Q. J. Roy. Meteorol. Soc., 143, 14591470, https://doi.org/10.1002/qj.3017, 2017.

Kidston, J., Scaife, A. A., Hardiman, S. C., Mitchell, D. M., Butchart, N., Baldwin, M. P., and Gray, L. J.: Strato- spheric influence on tropospheric jet streams, storm tracks and surface weather, Nat. Geosci., 8, 433-440, https://doi.org/10.1038/ngeo2424, 2015.

Kim, J. and Son, S.-W.: Tropical Cold-Point Tropopause: Climatology, Seasonal Cycle, and Intraseasonal Variability Derived from COSMIC GPS Radio Occultation Measurements, J. Clim., 25, 5343-5360, https://doi.org/10.1175/JCLI-D-11-00554.1, 2012.

Kohma, M. and Sato, K.: The effects of atmospheric waves on the amounts of polar stratospheric clouds, Atmos. Chem. Phys., 11, 11535-11552, https://doi.org/10.5194/acp-11-11535-2011, 2011.

Kornhuber, K., Petoukhov, V., Petri, S., Rahmstorf, S., and Coumou, D.: Evidence for wave resonance as a key mechanism for generating high-amplitude quasi-stationary waves in boreal summer, Clim. Dynam., 49, 1961-1979, https://doi.org/10.1007/s00382016-3399-6, 2017.

Kornhuber, K., Osprey, S., Coumou, D., Petri, S., Petoukhov, V., Rahmstorf, S., and Gray, L.: Extreme weather events in early summer 2018 connected by a recurrent hemispheric wave-7 pattern, Environ. Res. Lett., 14, 054002, https://doi.org/10.1088/1748-9326/ab13bf, 2019.

Kursinski, E. R., Hajj, G. A., Schofield, J. T., Linfield, R. P., and Hardy, K. R.: Observing Earth's atmosphere with radio occultation measurements using the Global Positioning System, J. Geophys. Res.-Atmos., 102, 23429-23465, https://doi.org/10.1029/97JD01569, 1997.

Lau, N.-C.: The Structure and Energetics of Transient Disturbances in the Northern Hemisphere Wintertime Circulation, J. Atmos. Sci., 36, 982-995, https://doi.org/10.1175/15200469(1979)036<0982:TSAEOT>2.0.CO;2, 1979.

Lorenz, D. J. and Hartmann, D. L.: Eddy-Zonal Flow Feedback in the Southern Hemisphere, J. Atmos. Sci., 58, 3312-3327, https://doi.org/10.1175/15200469(2001)058<3312:EZFFIT>2.0.CO;2, 2001.

Lorenz, D. J. and Hartmann, D. L.: Eddy-Zonal Flow Feedback in the Northern Hemisphere Winter, J. Clim., 16, 1212-1227, https://doi.org/10.1175/15200442(2003)16<1212:EFFITN>2.0.CO;2, 2003.

Madden, R. A. and Julian, P. R.: Observations of the 40 50-Day Tropical Oscillation - A Review, Mon. Weather Rev., 122, 814, https://doi.org/10.1175/15200493(1994)122<0814:OOTDTO>2.0.CO;2, 1994.

Madhavi, G. N., Kishore, P., Rao, S. V. B., Velicogna, I., and Basha, G.: Two-day wave observations over the middle and high latitudes in the $\mathrm{NH}$ and $\mathrm{SH}$ using COSMIC GPSRO measurements, Adv. Space Res., 55, 722-731, https://doi.org/10.1016/j.asr.2014.09.032, 2015.

Martínez-Alvarado, O., Madonna, E., Gray, S. L., and Joos, H.: A route to systematic error in forecasts of Rossby waves, Q. J. Roy. Meteorol. Soc., 142, 196-210, https://doi.org/10.1002/qj.2645, 2016.

Martius, O., Schwierz, C., and Davies, H. C.: TropopauseLevel Waveguides, J. Atmos. Sci., 67, 866-879, https://doi.org/10.1175/2009JAS2995.1, 2010.

Nielsen-Gammon, J. W. and Lefevre, R. J.: Piecewise Tendency Diagnosis of Dynamical Processes Governing the Development of an Upper-Tropospheric Mobile Trough, J. Atmos. Sci., 53, 3120-3142, https://doi.org/10.1175/15200469(1996)053<3120:PTDODP>2.0.CO;2, 1996. 
Oberländer-Hayn, S., Gerber, E. P., Abalichin, J., Akiyoshi, H., Kerschbaumer, A., Kubin, A., Kunze, M., Langematz, U., Meul, S., Michou, M., Morgenstern, O., and Oman, L. D.: Is the Brewer-Dobson circulation increasing or moving upward?, Geophys. Res. Lett., 43, 1772-1779, https://doi.org/10.1002/2015GL067545, 2016.

Odell, L., Knippertz, P., Pickering, S., Parkes, B., and Roberts, A.: The Braer storm revisited, Weather, 68, 105-111, https://doi.org/10.1002/wea.2097, 2013.

Orlanski, I. and Katzfey, J.: The Life Cycle of a Cyclone Wave in the Southern Hemisphere. Part I: Eddy Energy Budget, J. Atmos. Sci., 48, 1972-1998, https://doi.org/10.1175/15200469(1991)048<1972:TLCOAC $>2.0 . C O ; 2,1991$.

Orlanski, I. and Sheldon, J.: A Case of Downstream Baroclinic Development over Western North America, Mon. Weather Rev., 121, 2929, https://doi.org/10.1175/15200493(1993)121<2929:ACODBD>2.0.CO;2, 1993.

Petoukhov, V., Rahmstorf, S., Petri, S., and Schellnhuber, H. J.: Quasiresonant amplification of planetary waves and recent Northern Hemisphere weather extremes, P. Natl. Acad. Sci. USA, 110, 5336-5341, https://doi.org/10.1073/pnas.1222000110, 2013.

Pilch Kedzierski, R., Matthes, K., and Bumke, K.: The tropical tropopause inversion layer: variability and modulation by equatorial waves, Atmos. Chem. Phys., 16, 11617-11633, https://doi.org/10.5194/acp-16-11617-2016, 2016 .

Pilch Kedzierski, R., Neef, L., and Matthes, K.: Tropopause sharpening by data assimilation, Geophys. Res. Lett., 43, 8298-8305, https://doi.org/10.1002/2016GL069936, 2016b.

Pilch Kedzierski, R., Matthes, K., and Bumke, K.: Wave modulation of the extratropical tropopause inversion layer, Atmos. Chem. Phys., 17, 4093-4114, https://doi.org/10.5194/acp-174093-2017, 2017.

Pirret, J. S. R., Knippertz, P., and Trzeciak, T. M.: Drivers for the deepening of severe European windstorms and their impacts on forecast quality, Q. J. Roy. Meteorol. Soc., 143, 309-320, https://doi.org/10.1002/qj.2923, 2017.

Poli, P., Joiner, J., and Kursinski, E. R.: 1DVAR analysis of temperature and humidity using GPS radio occultation refractivity data, J. Geophys. Res.-Atmos., 107, ACL 14-1-ACL 14-20, https://doi.org/10.1029/2001JD000935, 2002.

Quinting, J. F. and Vitart, F.: Representation of Synoptic-Scale Rossby Wave Packets and Blocking in the S2S Prediction Project Database, Geophys. Res. Lett., 46, 1070-1078, https://doi.org/10.1029/2018GL081381, 2019.

Randel, W. J. and Stanford, J. L.: An Observational Study of Medium-Scale Wave Dynamics in the Southern Hemisphere Summer. Part I: Wave Structure and Energetics, J. Atmos. Sci., 42, 1172-1188, https://doi.org/10.1175/15200469(1985)042<1172:AOSOMS>2.0.CO;2, 1985.

Randel, W. J. and Wu, F.: Kelvin wave variability near the equatorial tropopause observed in GPS radio occultation measurements, J. Geophys. Res.-Atmos., 110, D03102, https://doi.org/10.1029/2004JD005006, 2005.

R Core Team: R: A Language and Environment for Statistical Computing, R Foundation for Statistical Computing, Vienna, Austria, available at: https://www.r-project.org/ (last access: 15 July 2020), 2015.
Rossby, C.-G.: On the Propagation of Frequencies and Energy in Certain Types of Oceanic and Atmospheric Waves, J. Atmos. Sci., 2, 187-204, https://doi.org/10.1175/15200469(1945)002<0187:OTPOFA>2.0.CO;2, 1945.

Saffin, L., Gray, S. L., Methven, J., and Williams, K. D.: Processes Maintaining Tropopause Sharpness in Numerical Models, J. Geophys. Res.-Atmos., 122, 9611-9627, https://doi.org/10.1002/2017JD026879, 2017.

Scaife, A. A., Spangehl, T., Fereday, D. R., Cubasch, U., Langematz, U., Akiyoshi, H., Bekki, S., Braesicke, P., Butchart, N., Chipperfield, M. P., Gettelman, A., Hardiman, S. C., Michou, M., Rozanov, E., and Shepherd, T. G.: Climate change projections and stratosphere-troposphere interaction, Clim. Dynam., 38, 2089-2097, https://doi.org/10.1007/s00382-011-10807, 2012.

Scherllin-Pirscher, B., Randel, W. J., and Kim, J.: Tropical temperature variability and Kelvin-wave activity in the UTLS from GPS RO measurements, Atmos. Chem. Phys., 17, 793-806, https://doi.org/10.5194/acp-17-793-2017, 2017.

Schmidt, T., Alexander, P., and de la Torre, A.: Stratospheric gravity wave momentum flux from radio occultations, J. Geophys. Res.-Atmos., 121, 4443-4467, https://doi.org/10.1002/2015JD024135, 2016.

Schreck, C.: Extract equatorial waves by filtering in the Wheeler-Kiladis wavenumber-frequency domain, NCAR, available at: https://www.ncl.ucar.edu/Document/Functions/User_ contributed/kf_filter.shtml (last access: 15 July 2020), 2009.

Schreiner, W., Sokolovskiy, S., Hunt, D., Rocken, C., and Kuo, Y.-H.: Analysis of GPS radio occultation data from the FORMOSAT-3/COSMIC and Metop/GRAS missions at CDAAC, Atmos. Meas. Tech., 4, 2255-2272, https://doi.org/10.5194/amt-4-2255-2011, 2011.

Sellwood, K. J., Majumdar, S. J., Mapes, B. E., and Szunyogh, I.: Predicting the influence of observations on medium-range forecasts of atmospheric flow, Q. J. Roy. Meteorol. Soc., 134, 20112027, https://doi.org/10.1002/qj.341, 2008.

Shapiro, M. A. and Keyser, D.: Extratropical Cyclones, in: The Erik Palmén Memorial Volume, Chapt. "Fronts, Jet Streams and the Tropopause", American Meteorological Society, Boston, MA, pp. 167-191, 1990.

Shaw, T. A., Baldwin, M., Barnes, E. A., Caballero, R., Garfinkel, C. I., Hwang, Y. T., Li, C., O’Gorman, P. A., Rivière, G., and Simpson, I. R.: Storm track processes and the opposing influences of climate change, Nat. Geosci., 9, 656-664, https://doi.org/10.1038/ngeo2783, 2016.

Shepherd, M. G. and Tsuda, T.: Large-scale planetary disturbances in stratospheric temperature at high-latitudes in the southern summer hemisphere, Atmos. Chem. Phys., 8, 7557-7570, https://doi.org/10.5194/acp-8-7557-2008, 2008.

Simmons, A. J. and Hoskins, B. J.: The Life Cycles of Some Nonlinear Baroclinic Waves, J. Atmos. Sci., 35, 414-432, https://doi.org/10.1175/15200469(1978)035<0414:TLCOSN>2.0.CO;2, 1978.

Son, S.-W., Tandon, N. F., and Polvani, L. M.: The fine-scale structure of the global tropopause derived from COSMIC GPS radio occultation measurements, J. Geophys. Res.-Atmos., 116, D20113, https://doi.org/10.1029/2011JD016030, 2011.

Souders, M. B., Colle, B. A., and Chang, E. K. M.: A Description and Evaluation of an Automated Approach for Feature- 
Based Tracking of Rossby Wave Packets, Mon. Weather Rev., 142, 3505-3527, https://doi.org/10.1175/MWR-D-13-00317.1, 2014a.

Souders, M. B., Colle, B. A., and Chang, E. K. M.: The Climatology and Characteristics of Rossby Wave Packets Using a FeatureBased Tracking Technique, Mon. Weather Rev., 142, 3528-3548, https://doi.org/10.1175/MWR-D-13-00371.1, 2014b.

Steiner, A. K., Ladstädter, F., Ao, C. O., Gleisner, H., Ho, S.-P., Hunt, D., Schmidt, T., Foelsche, U., Kirchengast, G., Kuo, Y.H., Lauritsen, K. B., Mannucci, A. J., Nielsen, J. K., Schreiner, W., Schwärz, M., Sokolovskiy, S., Syndergaard, S., and Wickert, J.: Consistency and structural uncertainty of multi-mission GPS radio occultation records, Atmos. Meas. Tech., 13, 2547-2575, https://doi.org/10.5194/amt-13-2547-2020, 2020.

Takaya, K. and Nakamura, H.: A Formulation of a PhaseIndependent Wave-Activity Flux for Stationary and Migratory Quasigeostrophic Eddies on a Zonally Varying Basic Flow, J. Atmos. Sci., 58, 608-627, https://doi.org/10.1175/15200469(2001)058<0608:AFOAPI>2.0.CO;2, 2001.

Tao, W., Zhang, J., Fu, Y., and Zhang, X.: Driving Roles of Tropospheric and Stratospheric Thermal Anomalies in Intensification and Persistence of the Arctic Superstorm in 2012, Geophys. Res. Lett., 44, 10, https://doi.org/10.1002/2017GL074778, 2017a.

Tao, W., Zhang, J., and Zhang, X.: The role of stratosphere vortex downward intrusion in a long-lasting late-summer Arctic storm, Q. J. Roy. Meteorol. Soc., 143, 1953-1966, https://doi.org/10.1002/qj.3055, 2017 b.

Teubler, F. and Riemer, M.: Dynamics of Rossby Wave Packets in a Quantitative Potential Vorticity-Potential Temperature Framework, J. Atmos. Sci., 73, 1063-1081, https://doi.org/10.1175/JAS-D-15-0162.1, 2016.

Thorncroft, C. D., Hoskins, B. J., and McIntyre, M. E.: Two paradigms of baroclinic-wave life-cycle behaviour, Q. J. Roy. Meteorol. Soc., 119, 17-55, https://doi.org/10.1002/qj.49711950903, 1993.

Tsuda, T.: Characteristics of atmospheric gravity waves observed using the MU (Middle and Upper atmosphere) radar and GPS (Global Positioning System) radio occultation, Proc. Jpn. Acad. Ser. B, 90, 12-27, https://doi.org/10.2183/pjab.90.12, 2014.

Tsuda, T., Nishida, M., Rocken, C., and Ware, R. H.: A Global Morphology of Gravity Wave Activity in the Stratosphere Revealed by the GPS Occultation Data (GPS/MET), J. Geophys. Res., 105, 7257-7273, https://doi.org/10.1029/1999JD901005, 2000.

von Engeln, A., Andres, Y., Marquardt, C., and Sancho, F.: GRAS radio occultation on-board of Metop, Adv. Space Res., 47, 336347, https://doi.org/10.1016/j.asr.2010.07.028, 2011.

Wang, L. and Alexander, M. J.: Global estimates of gravity wave parameters from GPS radio occultation temperature data, J. Geophys. Res.-Atmos., 115, D21122, https://doi.org/10.1029/2010JD013860, 2010.
Webster, P. J. and Keller, J. L.: Atmospheric Variations: Vacillations and Index Cycles, J. Atmos. Sci., 32, 1283-1301, https://doi.org/10.1175/15200469(1975)032<1283:AVVAIC>2.0.CO;2, 1975.

Wickert, J., Reigber, C., Beyerle, G., König, R., Marquardt, C., Schmidt, T., Grunwaldt, L., Galas, R., Meehan, T. K., Melbourne, W. G., and Hocke, K.: Atmosphere sounding by GPS radio occultation: First results from CHAMP, Geophys. Res. Lett., 28, 3263-3266, https://doi.org/10.1029/2001GL013117, 2001.

Wickert, J., Michalak, G., Schmidt, T., Beyerle, G., Cheng, C. Z., Healy, S. B., Heise, S., Huang, C. Y., Jakowski, N., Köhler, W., Mayer, C., Offiler, D., Ozawa, E., Pavelyev, A. G., Rothacher, M., Tapley, B., and Arras, C.: GPS radio occultation: results from CHAMP, GRACE and FORMOSAT-3/COSMIC, Terr. Atmos. Ocean. Sci., 20, 35-50, https://doi.org/10.3319/TAO.2007.12.26.01(F3C), 2009.

Williams, I. N. and Colucci, S. J.: Characteristics of Baroclinic Wave Packets during Strong and Weak Stratospheric Polar Vortex Events, J. Atmos. Sci., 67, 3190-3207, https://doi.org/10.1175/2010JAS3279.1, 2010.

Wirth, V., Riemer, M., Chang, E. K. M., and Martius, O.: Rossby Wave Packets on the Midlatitude Waveguide - A Review, Mon. Weather Rev., 146, 1965-2001, https://doi.org/10.1175/MWRD-16-0483.1, 2018.

WMO: Meteorology-A three-dimensional science, WMO Bull., 6 , 134-138, 1957.

Wolf, G. and Wirth, V.: Implications of the Semigeostrophic Nature of Rossby Waves for Rossby Wave Packet Detection, Mon. Weather Rev., 143, 26-38, https://doi.org/10.1175/MWR-D-1400120.1, 2015.

Wolf, G. and Wirth, V.: Diagnosing the Horizontal Propagation of Rossby Wave Packets along the Midlatitude Waveguide, Mon. Weather Rev., 145, 3247-3264, https://doi.org/10.1175/MWRD-16-0355.1, 2017.

Zeng, Z., Ho, S.-P., Sokolovskiy, S., and Kuo, Y.-H.: Structural evolution of the Madden-Julian Oscillation from COSMIC radio occultation data, J. Geophys. Res.-Atmos., 117, D22108, https://doi.org/10.1029/2012JD017685, 2012.

Zheng, M., Chang, E. K. M., and Colle, B. A.: Ensemble Sensitivity Tools for Assessing Extratropical Cyclone Intensity and Track Predictability, Weather Forecast., 28, 1133-1156, https://doi.org/10.1175/WAF-D-12-00132.1, 2013.

Zimin, A. V., Szunyogh, I., Patil, D. J., Hunt, B. R., and Ott, E.: Extracting Envelopes of Rossby Wave Packets, Mon. Weather Rev., 131, 1011, https://doi.org/10.1175/15200493(2003)131<1011:EEORWP>2.0.CO;2, 2003.

Zimin, A. V., Szunyogh, I., Hunt, B. R., and Ott, E.: Extracting Envelopes of Nonzonally Propagating Rossby Wave Packets, Mon. Weather Rev., 134, 1329, https://doi.org/10.1175/MWR3122.1, 2006. 